\title{
Modelagem computacional do trocador de calor solo-ar adaptado às condições climáticas de Belém
}

\author{
Computational modelling of an earth-air heat exchanger \\ (EAHE) adapted to the weather conditions of Belém, \\ Brazil
}

\section{Giovanni Antonio Vielma Vivas Danielle Regina da Silva Guerra}

\section{Resumo}

A demanda mundial de energia está em constante crescimento, ao mesmo tempo as reservas de combustíveis fósseis estão diminuindo e não serão suficientes para satisfazer as demandas globais de energia no futuro. Além disso, os sistemas convencionais de aquecimento e refrigeração consomem a maior parte da demanda por energia elevando consideravelmente os custos. $\mathrm{O}$ trocador de calor solo-ar (TCSA) é uma opção sustentável que consume menos energia com potencial para melhorar as condições térmicas em edifícios. Apesar de ser uma tecnologia simples e antiga, no Brasil as pesquisas sobre eles são muito limitadas. Nesse contexto, faltam estudos na Região Norte deste país, em particular na cidade de Belém do Pará. Portanto, o objetivo desta pesquisa é desenvolver um modelo numérico tridimensional do TCSA usando ANSYS Fluent para avaliar a influência de parâmetros operacionais e construtivos, tais como a velocidade de escoamento do ar, comprimento e diâmetro do duto no desempenho térmico desse sistema adaptado às condições climáticas e às características do solo dessa cidade. A partir dos resultados obtidos, conclui-se que esse sistema pode reduzir a temperatura do ar na saída do duto até em 5,91 ${ }^{\circ} \mathrm{C}$.

Palavras-chave: Trocador de calor solo-ar (TCSA). Resfriamento de edifícios. Simulação numérica. Energia renovável.

\begin{abstract}
The world's demand for energy is constantly increasing, while, at the same time, the reserves of fossil fuels are decreasing and will not be enough to satisfy the global energy demand in the future. In addition, conventional heating and cooling systems consume most of the energy demand and considerably increase costs. Earth-air heat exchangers (EAHE) are a sustainable option that consumes less energy and has the potential to improve thermal conditions in buildings. Despite it being a simple and old technology, research into this type of technology is very limited in Brazil. There is a lack of studies in the northern region of this country, particularly in the city of Belém do Pará. Hence, the goal of this research study was to develop a threedimensional numerical model of EAHE using ANSYS Fluent to evaluate the influence of operational and constructive parameters such as air flow velocity, length and diameter of the duct on the thermal performance of this system adapted to climatic conditions and soil characteristics of this city. The results obtained lead to the conclusion that this system can reduce the air temperature at the duct outlet up to $5.91{ }^{\circ} \mathrm{C}$.
\end{abstract}

Recebido em 12/01/20

Aceito em 31/03/20

Keywords: Earth-Air Heat Exchangers (EAHE). Building cooling. Numerical simulation. Renewable energy. 


\section{Introdução}

A demanda por energia está crescendo diariamente em todo o mundo e as reservas de combustíveis fósseis não serão suficientes para satisfazer as demandas globais de energia no futuro (AGRAWAL et al., 2019a). A necessidade de energia para refrigeração e aquecimento do espaço representa cerca de um terço da demanda total de energia (INTERNATIONAL..., 2013). Espera-se que o número de sistemas de ar condicionado de edifícios aumente de 1,6 bilhão para 5,6 bilhões até 2050, o que aumentará a necessidade de energia para o condicionamento predial em quase três vezes (INTERNATIONAL..., 2018).

Os sistemas convencionais de aquecimento e refrigeração consomem muita energia e o custo da energia baseada em combustíveis fósseis aumenta continuamente (AGRAWAL et al., 2019a). Todas essas preocupações aumentaram o interesse dos pesquisadores e cientistas na utilização de sistemas baseados em energia renovável em larga escala para aquecimento e resfriamento do edifício. Os sistemas renováveis e passivos podem reduzir a demanda de energia de refrigeração e aquecimento do edifício. $\mathrm{O}$ trocador de calor solo-ar (TCSA) é um sistema promissor para fornecer efeitos de aquecimento/resfriamento e inclui os benefícios de menor consumo de energia e menor emissão de gases de efeito estufa (AGRAWAL et al., 2019a).

As medições mostram que para sistemas geotérmicos rasos a temperatura do solo abaixo de certa profundidade permanece relativamente constante ao longo do ano. Isso ocorre porque as flutuações de temperatura na superfície são diminuídas à medida que a profundidade do solo aumenta devido à alta inércia térmica do solo (KALOGIROU, 2014). De acordo com Popiel, Wojtkowiak e Biernacka (2001), do ponto de vista da distribuição de temperatura três zonas de solo podem ser distinguidas:

(a) zona de superfície, atingindo uma profundidade de cerca de $1 \mathrm{~m}$, na qual a temperatura do solo é muito sensível a mudanças de tempo curtas das condições climáticas;

(b) zona rasa, que se estende da profundidade de cerca de $1 \mathrm{~m}$ a $8 \mathrm{~m}$ (para solos secos e leves) ou $20 \mathrm{~m}$ (para solos úmidos, pesados e arenosos), onde a temperatura do solo é quase constante e próxima da temperatura média anual do ar (nessa zona as distribuições de temperatura do solo dependem principalmente das condições climáticas do ciclo sazonal); e

(c) zona profunda, abaixo da profundidade da zona rasa, onde a temperatura do solo é praticamente constante (e aumenta muito lentamente com a profundidade de acordo com o gradiente geotérmico).

Essa temperatura quase constante durante todo o ano é significativamente mais baixa no verão e mais alta no inverno do que a temperatura do ar ambiente (MISRA et al., 2013a, 2013b). Essa diferença de temperatura pode ser empregada para resfriar/aquecer o ar ambiente no verão/inverno, usando o trocador de calor solo-ar (BHARADWAJ; BANSAL, 1981; BISONIYA; KUMAR; BAREDAR, 2013; JACOVIDES et al., 1996).

O sistema TCSA utiliza o solo como fonte ou sumidouro de calor e o ar como meio de transferência de calor. Em um sistema TCSA típico, o ar externo é passado através dos tubos enterrados usando um soprador. Durante o escoamento do ar, ele troca o calor com o solo ao redor e é aquecido ou resfriado, dependendo das condições do ambiente (AGRAWAL et al., 2019a).

O desempenho do TCSA aumenta com a profundidade do tubo, mas o investimento inicial também aumenta (AGRAWAL et al., 2019a). De $2 \mathrm{~m}$ a $4 \mathrm{~m}$ de profundidade a temperatura do solo tende a ser afetada pelas propriedades do solo e pelas condições ambientais, como temperatura ambiente e chuva (LIU et al., 2019). Isso pode levar a uma grande variação da temperatura do ar de saída dos sistemas TCSA, diminuindo assim seu desempenho térmico (OMER, 2008). O princípio de funcionamento desse sistema para o resfriamento no verão é mostrado na Figura 1.

O princípio de funcionamento do TCSA é bem conhecido há muito tempo, mas geralmente não é usado em todos os países. Particularmente no Brasil, o número de trabalhos científicos sobre o tema do TCSA ainda é pequeno, com um interesse crescente nesta década (RODRIGUES et al., 2018). Por exemplo, uma avaliação sintética do potencial subterrâneo que cobre as oito zonas bioclimáticas desse país, de norte a sul, foi realizada em Alves e Schmid (2015). Além disso, Estrada et al. (2018) estudaram o potencial de resfriamento/aquecimento de diferentes regiões climáticas do Brasil, em comparação com o sul da França, considerando as trocas de calor sensível e latente. 
Figura 1 - Princípio de funcionamento do sistema TCSA para refrigeração no verão

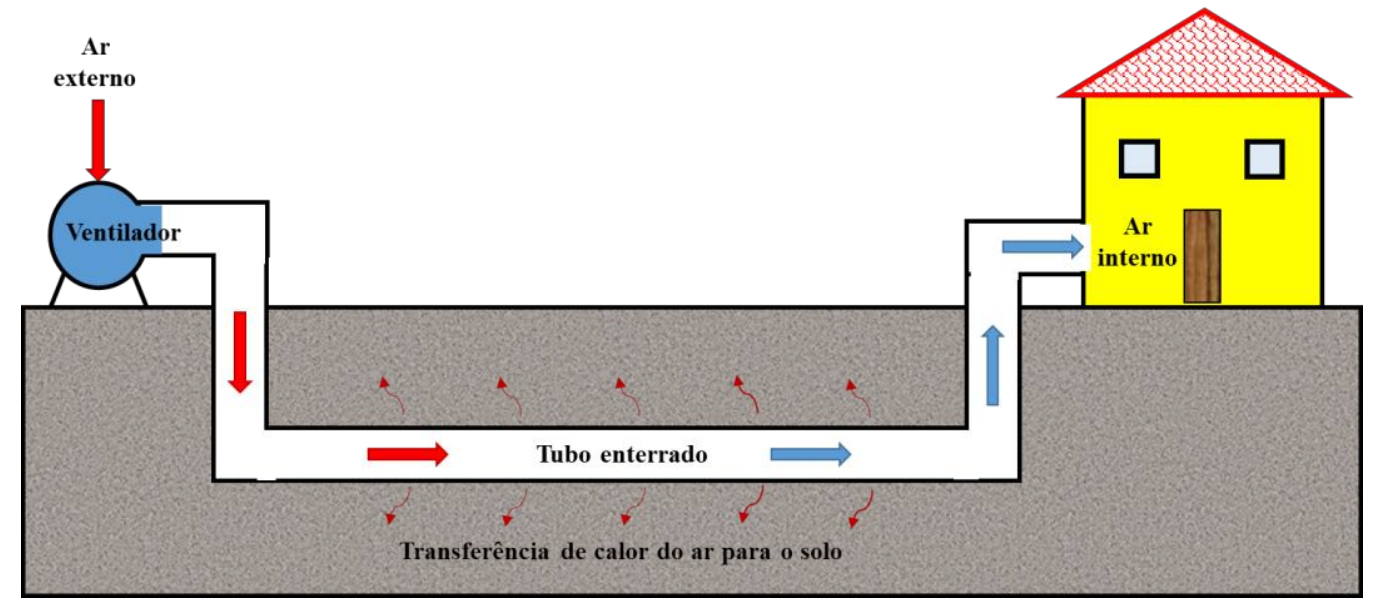

Na Região Sul, alto potencial térmico foi encontrado para o estado do Rio Grande do Sul nas obras de Vaz et al. (2011, 2014). Eles analisaram uma instalação experimental e relataram as temperaturas e propriedades do ar e solo locais medidas ao longo de um ano. Explorando esses dados, novos modelos completos para TCSA foram desenvolvidos por Brum et al. $(2012,2013)$ para estudar seu desempenho devido à profundidade dos dutos. Utilizando esses modelos, trabalhos recentes focaram no design das instalações (NUNES et al., 2015; RODRIGUES et al., 2015; BRUM et al., 2016a, 2016b, 2018; RAMALHO et al., 2018).

Em relação à influência do tipo de solo no desempenho térmico do TCSA, Rodrigues et al. (2018) estabeleceram que elevadas melhorias relativas (de mais de 60\%) são notadas quando o TCSA é instalado em camadas argilosas adequadas do solo. Esses autores apontam que é muito importante realizar testes in situ e simulações numéricas nos locais antes das instalações do TCSA, pois eles podem apresentar desempenhos muito ruins em alguns casos. Assim, é importante conhecer o perfil geotécnico do solo para permitir a construção de TCSA adequados. Outro trabalho relacionado a esse aspecto foi realizado por Rodrigues et al. (2017), que constataram que o TCSA, quando instalado em solo com característica argilosa, apresenta melhor desempenho térmico se comparado a uma instalação em solo arenoso.

Nesse contexto faltam estudos no Brasil considerando sua Região Norte, em particular na cidade de Belém de Pará. Na avaliação das oito zonas bioclimáticas do Brasil realizada por Alves e Schmid (2015), os pesquisadores concluíram que a cidade de Belém tem um significativo potencial de climatização subterrâneo. Eles recomendam um estudo mais detalhado sobre a aplicação de TCSA em cada zona apresentada, levando em consideração entre outras coisas as propriedades específicas do solo de cada região.

Em um estudo realizado por Oliveira (2018) no Campus Guamá da Universidade Federal do Pará (UFPA) na cidade de Belém, constatou-se que em um dos sítios onde foram feitos os ensaios tem-se solo do tipo argila siltosa até 22,20 $\mathrm{m}$ de profundidade. Além disso, ele também observou que se tem valores de nível de água na faixa entre $4 \mathrm{~cm}$ e $113 \mathrm{~cm}$, com uma média de $50 \mathrm{~cm}$ de profundidade na área de estudo. Essa característica dos solos da cidade de Belém pode ser benéfica para o desempenho do TCSA, conforme estabelecido por numerosos trabalhos que estudam a influência da umidade do solo na eficiência do TCSA (AGRAWAL et al., 2018, 2019b; CUNY et al., 2018, 2020; LI et al., 2019; LIN et al., 2020; MORSHED et al., 2018).

Em conformidade com o exposto até aqui nesta pesquisa, propõe-se desenvolver um modelo numérico tridimensional de TCSA usando o software ANSYS Fluent para avaliar a influência de parâmetros operacionais e construtivos tais como: velocidade de escoamento do ar e comprimento e diâmetro do duto no desempenho térmico desse sistema adaptado às condições climáticas e às características do solo da cidade de Belém.

\section{Método}

\section{Modelo físico}

Como mostra a Figura 2, neste estudo é assumido que o TCSA esteja enterrado a $3 \mathrm{~m}$ da superfície do solo na cidade de Belém do Pará. Em particular, o tipo de solo e seu teor de umidade foram estabelecidos por 
Oliveira (2018), que realizou ensaios no Parque de Ciência e Tecnologia do Guamá (PCT Guamá), que se encontra dentro da Universidade Federal do Pará, delimitada pelas coordenadas geográficas: $1^{\circ} 28^{\prime} 33,6^{\prime}$ ' e $1^{\circ} 27^{\prime} 45,8^{\prime}$ ' S de latitude, $48^{\circ} 27^{\prime} 34^{\prime}$ ' e $48^{\circ} 26^{\prime} 33,4^{\prime}$ ' $\mathrm{W}$ de longitude. Portanto, os dados de temperatura do ar exterior e do solo da cidade de Belém são empregados para avaliar o desempenho de resfriamento do TCSA proposto.

Além disso, para investigar a capacidade de resfriamento desse sistema, como em Zhou et al. (2018), as seguintes premissas são empregadas:

(a) o solo é um sólido isotrópico e homogêneo e seus parâmetros termofísicos são assumidos como constantes;

(b) o ar é incompressível e possui parâmetros termofísicos constantes;

(c) não são consideradas resistências térmicas de contato entre diferentes camadas de material;

(d) a espessura da parede do tubo é negligenciada;

(e) a condensação e a evaporação no interior do tubo e no solo são negligenciadas; e

(f) o efeito da gravidade é negligenciado.

\section{Modelo numérico}

O modelo numérico tridimensional para o estudo do TCSA é estabelecido usando o código de dinâmica dos fluídos computacional (ou CFD, do inglês computational fluid dynamics) comercial, ANSYS Fluent Student 2019 R2. Os detalhes desse modelo são fornecidos abaixo.

\section{Equações}

Para o ar, o modelo RNG k- $\varepsilon$ é escolhido para simular o processo turbulento de ar no tubo (sem termos fonte). De acordo com Versteeg e Malalasekera (2007), as equações de transporte que regem as coordenadas cartesianas 3D são:

Equação de continuidade (Equação 1):

$\frac{\partial u}{\partial x}+\frac{\partial v}{\partial y}+\frac{\partial w}{\partial z}=0$

Eq. 1

Equações de quantidade de movimento (Equações 2, 3 e 4):

$\rho_{a}\left(\frac{\partial u}{\partial t}+u \frac{\partial u}{\partial x}+v \frac{\partial u}{\partial y}+w \frac{\partial u}{\partial z}\right)=-\frac{\partial p}{\partial x}+\mu_{a}\left(\frac{\partial^{2} u}{\partial x^{2}}+\frac{\partial^{2} u}{\partial y^{2}}+\frac{\partial^{2} u}{\partial z^{2}}\right)$

$\rho_{a}\left(\frac{\partial v}{\partial t}+u \frac{\partial v}{\partial x}+v \frac{\partial v}{\partial y}+w \frac{\partial v}{\partial z}\right)=-\frac{\partial p}{\partial y}+\mu_{a}\left(\frac{\partial^{2} v}{\partial x^{2}}+\frac{\partial^{2} v}{\partial y^{2}}+\frac{\partial^{2} v}{\partial z^{2}}\right)$

Eq. 3

$\rho_{a}\left(\frac{\partial w}{\partial t}+u \frac{\partial w}{\partial x}+v \frac{\partial w}{\partial y}+w \frac{\partial w}{\partial z}\right)=-\frac{\partial p}{\partial z}+\mu_{a}\left(\frac{\partial^{2} w}{\partial x^{2}}+\frac{\partial^{2} w}{\partial y^{2}}+\frac{\partial^{2} w}{\partial z^{2}}\right)$

Eq. 4

Equação de energia (Equação 5):

$\rho_{a} C_{a}\left(\frac{\partial T}{\partial t}+u \frac{\partial T}{\partial x}+v \frac{\partial T}{\partial y}+w \frac{\partial T}{\partial z}\right)=\frac{\partial}{\partial x}\left(\lambda_{a} \frac{\partial T}{\partial x}\right)+\frac{\partial}{\partial y}\left(\lambda_{a} \frac{\partial T}{\partial y}\right)+\frac{\partial}{\partial z}\left(\lambda_{a} \frac{\partial T}{\partial z}\right)$

Figura 2 - 0 diagrama esquemático do sistema TCSA

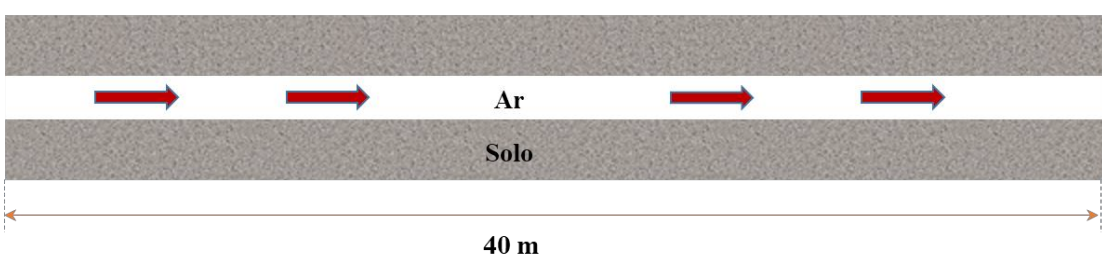

(a) Vista lateral

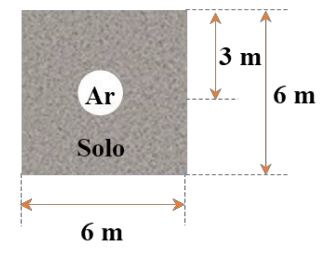

(b) Vista frontal 
Equações de transporte para o modelo RNG k-E (Equações 6, 7, 8 e 9):

$\frac{\partial\left(\rho_{a} k\right)}{\partial t}+\frac{\partial\left(\rho_{a} k u\right)}{\partial x}+\frac{\partial\left(\rho_{a} k v\right)}{\partial y}+\frac{\partial\left(\rho_{a} k w\right)}{\partial z}=\frac{\partial}{\partial x}\left(\frac{\mu_{t}}{\sigma_{k}} \frac{\partial k}{\partial x}\right)+\frac{\partial}{\partial y}\left(\frac{\mu_{t}}{\sigma_{k}} \frac{\partial k}{\partial y}\right)+\frac{\partial}{\partial z}\left(\frac{\mu_{t}}{\sigma_{k}} \frac{\partial k}{\partial z}\right)+G_{k}-\rho_{a} \varepsilon$ Eq. 6

$\frac{\partial\left(\rho_{a} \varepsilon\right)}{\partial t}+\frac{\partial\left(\rho_{a} \varepsilon u\right)}{\partial x}+\frac{\partial\left(\rho_{a} \varepsilon v\right)}{\partial y}+\frac{\partial\left(\rho_{a} \varepsilon w\right)}{\partial z}=\frac{\partial}{\partial x}\left(\frac{\mu_{t}}{\sigma_{\varepsilon}} \frac{\partial \varepsilon}{\partial x}\right)+\frac{\partial}{\partial y}\left(\frac{\mu_{t}}{\sigma_{\varepsilon}} \frac{\partial \varepsilon}{\partial y}\right)+\frac{\partial}{\partial z}\left(\frac{\mu_{t}}{\sigma_{\varepsilon}} \frac{\partial \varepsilon}{\partial z}\right)+C_{1 \varepsilon} \frac{\varepsilon}{k} G_{k}-C_{2 \varepsilon} \rho_{a} \frac{\varepsilon^{2}}{k}$

Onde:

$\mu_{t}=\rho_{a} C_{\mu} \frac{k^{2}}{\varepsilon}$

Eq. 8

e

$G_{k}=2 \mu_{t} E_{i j} \cdot E_{i j}$

Eq. 9

Nas Equações de 1 a $9, u(\mathrm{~m} / \mathrm{s}), v(\mathrm{~m} / \mathrm{s})$ e $w(\mathrm{~m} / \mathrm{s})$ são componentes de velocidade do fluido; $x(\mathrm{~m}) y(\mathrm{~m})$ e $z$ (m) são componentes de comprimento; $t$ (s) é o tempo; $\rho_{a}\left(\mathrm{~kg} / \mathrm{m}^{3}\right)$ é a massa específica do ar; $p(\mathrm{~Pa})$ é a pressão; $C_{a}\left(\mathrm{~J} / \mathrm{kg}^{\circ} \mathrm{C}\right)$ é o calor específico do ar; $T\left({ }^{\circ} \mathrm{C}\right)$ é a temperatura; $\lambda_{a}\left(\mathrm{~W} / \mathrm{m}^{\circ} \mathrm{C}\right)$ é a condutividade térmica do ar; $\mu_{\mathrm{a}}$ (Pa.s) é a viscosidade do ar, $k\left(\mathrm{~m}^{2} / \mathrm{s}^{2}\right)$ é a energia cinética turbulenta; $\varepsilon\left(\mathrm{m}^{2} / \mathrm{s}^{3}\right)$ é a taxa de dissipação de energia turbulenta; $\mu_{\mathrm{t}}\left(\mathrm{m}^{2} / \mathrm{s}\right)$ é a viscosidade turbulenta (ou $\left.e d d y\right)$ do ar; $G_{\mathrm{k}}\left(\mathrm{W} / \mathrm{m}^{3}\right)$ representa a geração de energia cinética de turbulência devido aos gradientes médios de velocidade; $C_{1 \varepsilon}, C_{2 \varepsilon}, C_{\mu}, \sigma_{\varepsilon}$ e $\sigma_{k}$ são constantes. Para o solo, apenas o processo de condução de calor é considerado (Equação 10):

$\rho_{s} C_{s} \frac{\partial T}{\partial t}=\frac{\partial}{\partial x}\left(\lambda_{s} \frac{\partial T}{\partial x}\right)+\frac{\partial}{\partial y}\left(\lambda_{s} \frac{\partial T}{\partial y}\right)+\frac{\partial}{\partial z}\left(\lambda_{s} \frac{\partial T}{\partial z}\right)$

Eq. 10

Onde:

$\rho_{s}\left(\mathrm{~kg} / \mathrm{m}^{3}\right)$ é a massa específica do solo;

$C_{s}\left(\mathrm{~J} / \mathrm{kg}^{\circ} \mathrm{C}\right)$ é o calor específico do solo; e

$\lambda_{s}\left(\mathrm{~W} / \mathrm{m}^{\circ} \mathrm{C}\right)$ é a condutividade térmica do solo.

\section{Geometria e materiais}

A Figura 3 mostra as dimensões geométricas do domínio computacional. Este tem comprimento de $40 \mathrm{~m}$ e largura e altura de $6 \mathrm{~m}$. O tubo é enterrado a uma profundidade de $3 \mathrm{~m}$. Em relação ao diâmetro, três diâmetros foram considerados, $0,2 \mathrm{~m}, 0,3 \mathrm{~m}$ e $0,4 \mathrm{~m}$. Além disso, a Tabela 1 lista os parâmetros termofísicos utilizados neste estudo.

\section{Seleção do dia de estudo}

A partir dos dados do Instituto Nacional de Meteorologia do Brasil (INMET) obtidos através do site do Laboratório de Eficiência Energética em Edificações (LABEEE) (LABORATÓRIO..., 2019) da Universidade Federal de Santa Catarina (UFSCA), foi determinada a temperatura máxima do ar externo da cidade de Belém, para cada dia do ano de 2010. Além disso, com as coordenadas globais dessa cidade, a temperatura do solo a uma profundidade de $2,89 \mathrm{~m}$ foi obtida do site do Centro Europeu de Previsão Meteorológica (ou ECMWF, do inglês European Centre for Medium-Range Weather Forecasts), (EUROPEAN..., 2019), em quatro momentos diferentes, 0h, 6h, 12h e 18h, de cada dia do ano de 2010, com os quais foi determinada a média aritmética da temperatura do solo nessa profundidade, para cada mês desse ano. Então, com esses dados, a diferença entre a temperatura máxima do ar externo e a temperatura do solo em 2,89 m foi determinada para cada dia do ano de 2010, usando a temperatura média do solo do mês correspondente a cada dia. Essas diferenças são mostradas nas Figuras 4 e 5. 
Figura 3 - Dimensões geométricas do domínio computacional

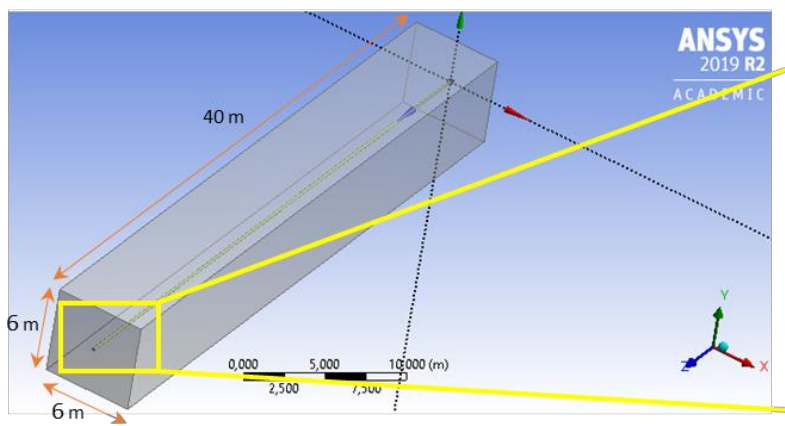

(a) Geometria completa

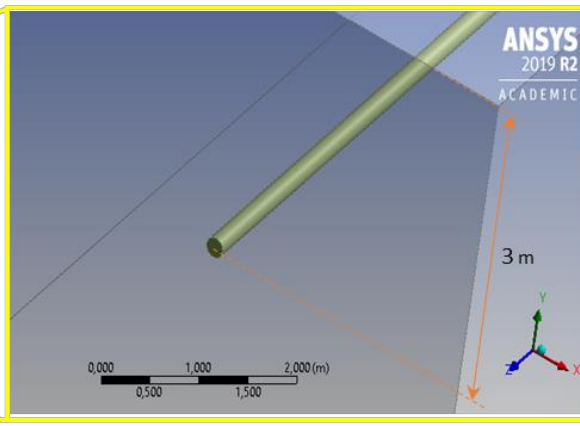

(b) Detalhe do duto

Tabela 1 - Propriedades termofísicas dos materiais utilizados

\begin{tabular}{c|c|c|c|c}
\hline Material & $\begin{array}{c}\text { Massa } \\
\text { específica } \\
\left(\mathbf{k g} / \mathbf{m}^{3}\right)\end{array}$ & $\begin{array}{c}\text { Calor } \\
\text { específico } \\
\left(\mathbf{J} /\left(\mathbf{k g}^{\circ} \mathbf{C}\right)\right)\end{array}$ & $\begin{array}{c}\text { Condutividade } \\
\text { térmica } \\
\left(\mathbf{W} /\left(\mathbf{m}^{\circ} \mathbf{C}\right)\right)\end{array}$ & $\begin{array}{c}\text { Viscosidade } \\
\text { dinâmica } \\
(\mathbf{k g} /(\mathbf{m} \cdot \mathbf{s}))\end{array}$ \\
\hline $\mathrm{Ar}^{1}$ & 1,165 & 1005 & 0,0267 & $1,90 \times 10^{-5}$ \\
Solo argiloso siltoso com $50 \%$ de umidade ${ }^{2}$ & 1120 & 2500 & 1,1 & \\
\hline
\end{tabular}

Nota: ${ }^{1}$ as propriedades termofísicas do ar foram obtidas do trabalho de Zhou et al. (2018); ${ }^{2}$ as propriedades termofísicas do solo foram fornecidas por Issa, Leitch e Chang (2015).

Figura 4 - Diferenças entre a temperatura diária máxima do ar externo e a temperatura média mensal do solo em 2,89 m para os meses de janeiro, fevereiro, março, abril, maio e junho

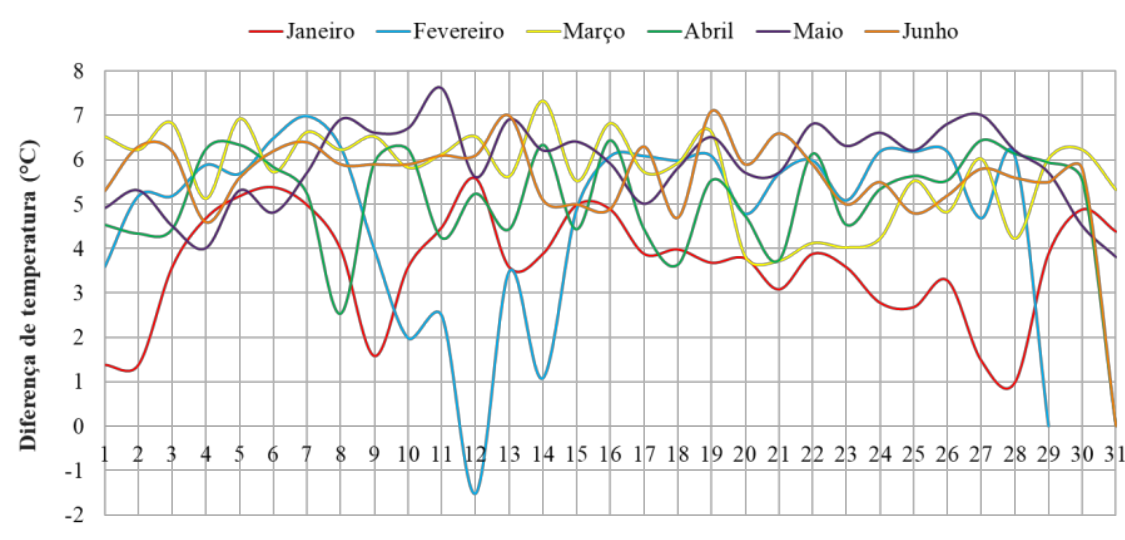

Dia

Figura 5 - Diferenças entre a temperatura diária máxima do ar externo e a temperatura média mensal do solo em 2,89 m para os meses de julho, agosto, setembro, outubro, novembro e dezembro

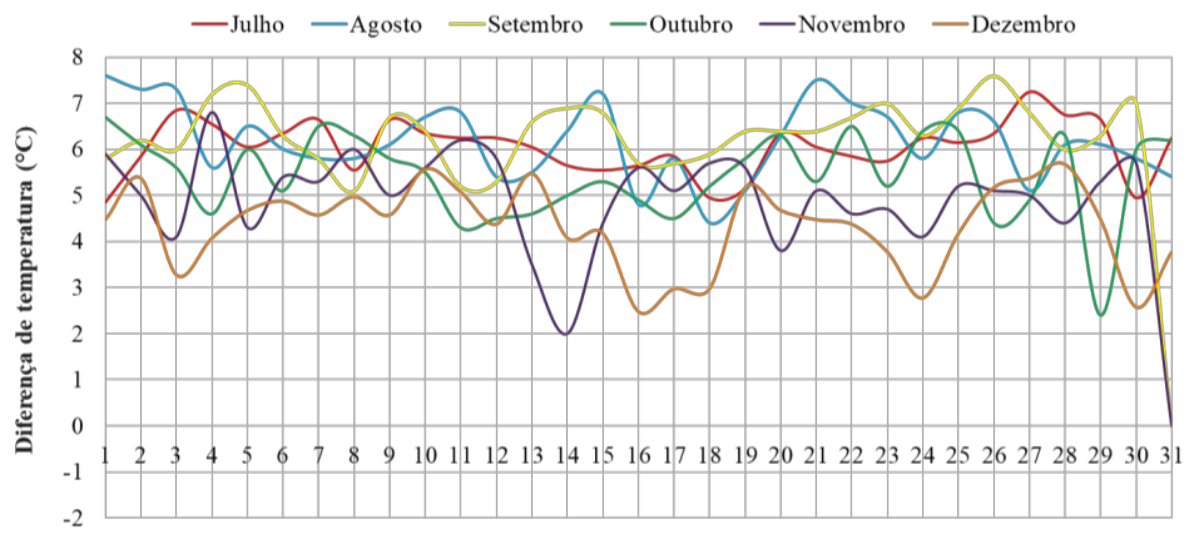

Dia 
A média aritmética dessas diferenças para os meses de janeiro, fevereiro, março, abril, maio e junho, julho, agosto, setembro, outubro, novembro e dezembro são $3,66{ }^{\circ} \mathrm{C}, 4,88^{\circ} \mathrm{C}, 5,71{ }^{\circ} \mathrm{C}, 5,20{ }^{\circ} \mathrm{C}, 5,88^{\circ} \mathrm{C}, 5,73{ }^{\circ} \mathrm{C}$, $6,05{ }^{\circ} \mathrm{C}, 6,18{ }^{\circ} \mathrm{C}, 6,35^{\circ} \mathrm{C}, 5,44^{\circ} \mathrm{C}, 5,01{ }^{\circ} \mathrm{C}, 4,34{ }^{\circ} \mathrm{C}$, respectivamente. Além disso, a média anual é de 5,37 ${ }^{\circ} \mathrm{C}$. Como mostram esses resultados, o mês de outubro é o de valor médio, mais próximo da média anual. Por esse motivo, esse mês foi selecionado para realizar o estudo do TCSA na cidade de Belém. Por outro lado, foi escolhido o dia $1^{\circ}$ desse mês, porque é aquele em que há maior diferença de temperatura, ou seja, $6,7{ }^{\circ} \mathrm{C}$. Nessa primeira investigação optou-se por estudar apenas um dia do ano, a fim de testar a metodologia utilizada. É importante observar que, para os meses do ano em que o TCSA tem maior potencial de uso na cidade de Belém, a média mensal da diferença de temperatura não é muito menor que a diferença correspondente ao primeiro de outubro, ou seja, 94,80\%, 92,17\%, 90,32\%, 87,73\%, 85,59\% e $85,15 \%$. Destes, $6,7^{\circ} \mathrm{C}$ para os meses de setembro, agosto, julho, maio, junho e março, respectivamente.

Embora o $1^{\circ}$ de outubro tenha sido o dia escolhido para analisar o desempenho do TCSA, nesta pesquisa as simulações foram realizadas em um intervalo de dois dias. O primeiro dia de simulação é necessário para que a temperatura do solo se estabilize, sendo então as análises realizadas com os dados do segundo dia de simulação. Portanto, os dados de temperatura externa do ar e do solo foram considerados para 30 de setembro e $1^{\circ}$ de outubro.

\section{Condições de fronteira}

\section{Entrada de ar}

Medições de temperatura horária do ar exterior de 30 de setembro e $1^{\circ}$ de outubro de 2010 da cidade de Belém realizadas pelo INMET foram obtidas através do site do LABEEE (LABORATÓRIO..., 2019) da UFSCA. Esses dados foram ajustados através de 6 splines cúbicos, usando o software estadístico gratuito R (THE R..., 2020), para obter a função de temperatura que foi usada como condição de contorno na entrada da tubulação. Essa função é dada pela Equação 11:

$$
T(t)=\left\{\begin{array}{c}
24,85+0,123 t-0,435 t^{2}+0,069 t^{3}, 0 \leq t<4 \\
31,097-4,562 t+0,736 t^{2}-0,029 t^{3}, 4 \leq t<12 \\
-46,687+14,884 t-0,885 t^{2}+0,016 t^{3}, 12 \leq t<18 \\
56,485-1,407 t-0,027 t^{2}+0,001 t^{3}, 18 \leq t<33 \\
986,370-85,942 t+2,534 t^{2}-0,025 t^{3}, 33 \leq t<39 \\
-2307,059+167,399 t-3,962 t^{2}+0,031 t^{3}, 39 \leq t \leq 48
\end{array}\right.
$$

Onde:

$t(h)$ é o tempo; e

$T\left({ }^{\circ} \mathrm{C}\right)$ é a temperatura.

Os valores de temperatura obtidos juntamente com as curvas de ajuste são mostrados na Figura 6. Essa função é definida no software ANSYS Fluent através de uma função definida pelo usuário (ou UDF, do inglês user defined function) desenvolvida na linguagem de programação C. Além disso, uma velocidade uniforme com uma direção normal para a fronteira é atribuída nessa entrada. Nesta pesquisa foram consideradas três velocidades, sendo $2 \mathrm{~m} / \mathrm{s}, 1,25 \mathrm{~m} / \mathrm{s}$ e $0,5 \mathrm{~m} / \mathrm{s}$.

\section{Saída de ar}

Como no trabalho de Rodrigues et al. (2018), na saída o ar é assumido à pressão atmosférica.

\section{Superfície do solo}

$\mathrm{Na}$ fronteira superior do domínio computacional foi considerada a temperatura do solo de 30 de setembro e $1^{\circ}$ de outubro de 2010 da cidade de Belém, obtida do ECMWF através de seu modelo ERA-Interim/Land (EUROPEAN..., 2019). O sistema integrado de previsão do ECMWF possui uma representação em quatro camadas do solo, onde a superfície está em $0 \mathrm{~m}$ e $0-0,07 \mathrm{~m}, 0,07-0,28 \mathrm{~m}, 0,28 \mathrm{~m}-1 \mathrm{~m}$ e $1-2,89 \mathrm{~m}$, são as camadas 1, 2, 3 e 4, respectivamente. A temperatura do solo é definida no meio de cada camada e a transferência de calor é calculada nas interfaces entre elas. Supõe-se que não exista transferência de calor do fundo da camada mais baixa. Essa plataforma oferece temperaturas do solo em quatro momentos diferentes do dia, 0h, 6h, 12h e 18h, para qualquer dia de 1979 a 2010. Nesse caso, os dados de temperatura da camada 
1 foram coletados para os dias já mencionados anteriormente e ajustados através de 6 splines cúbicos, novamente usando o software estadístico R (THE R..., 2020). Essa função é dada pela Equação 12:

$$
T(t)=\left\{\begin{array}{c}
26,25-1,684 t+0,045 t^{3}, 0 \leq t<3 \\
28,179-3,613 t+0,643 t^{2}-0,026 t^{3}, 3 \leq t<9 \\
12,077+1,754 t+0,047 t^{2}-0,004 t^{3}, 9 \leq t<15 \\
-25,194+9,208 t-0,450 t^{2}+0,007 t^{3}, 15 \leq t<33 \\
1353,704-116,146 t+3,348 t^{2}-0,032 t^{3}, 33 \leq t<40 \\
-3982,249+284,050 t-6,657 t^{2}+0,052 t^{3}, 40 \leq t \leq 48
\end{array}\right.
$$

Eq. 12

Onde:

$t(h)$ é o tempo; e

$T\left({ }^{\circ} \mathrm{C}\right)$ é a temperatura.

Os valores de temperatura obtidos juntamente com as curvas de ajuste são mostrados na Figura 7. Essa função também é definida no software ANSYS Fluent através de uma UDF.

Figura 6 - Dados de temperatura do ar na entrada do duto de 30 de setembro e $1^{\circ}$ de outubro de 2010 da cidade de Belém e o polinômio de ajuste correspondente

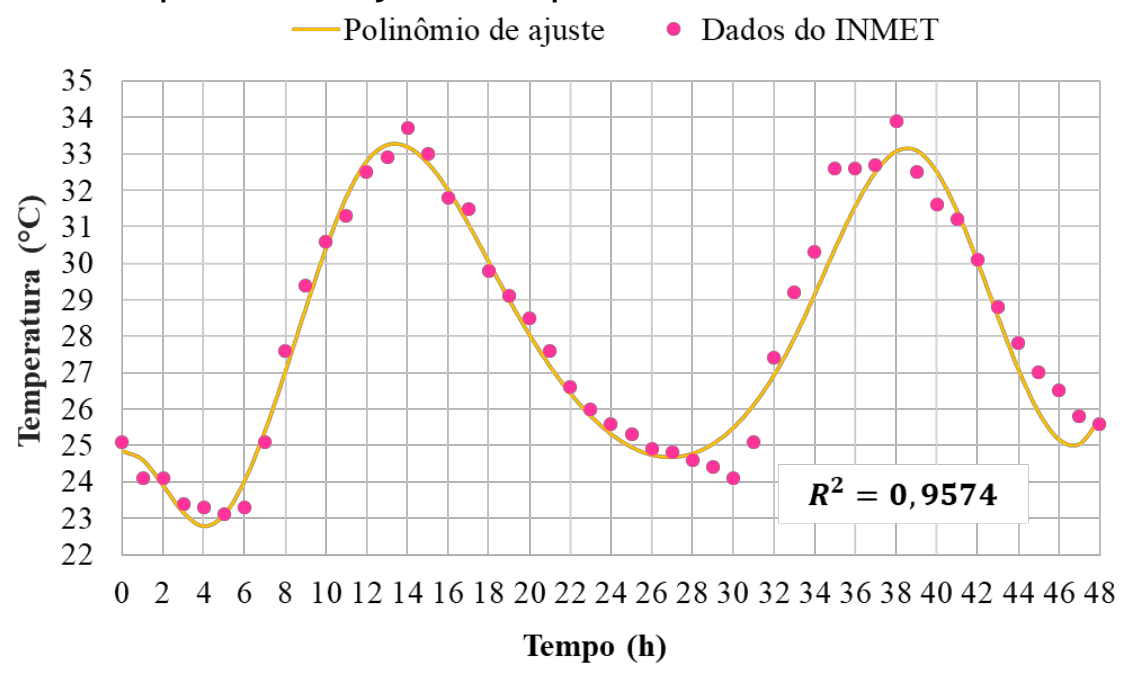

Fonte: Laboratório de Eficiência Energética em Edificações (2019).

Figura 7 - Dados de temperatura na superfície de solo de 30 de setembro e $1^{\circ}$ de outubro de 2010 da cidade de Belém e o polinômio de ajuste correspondente

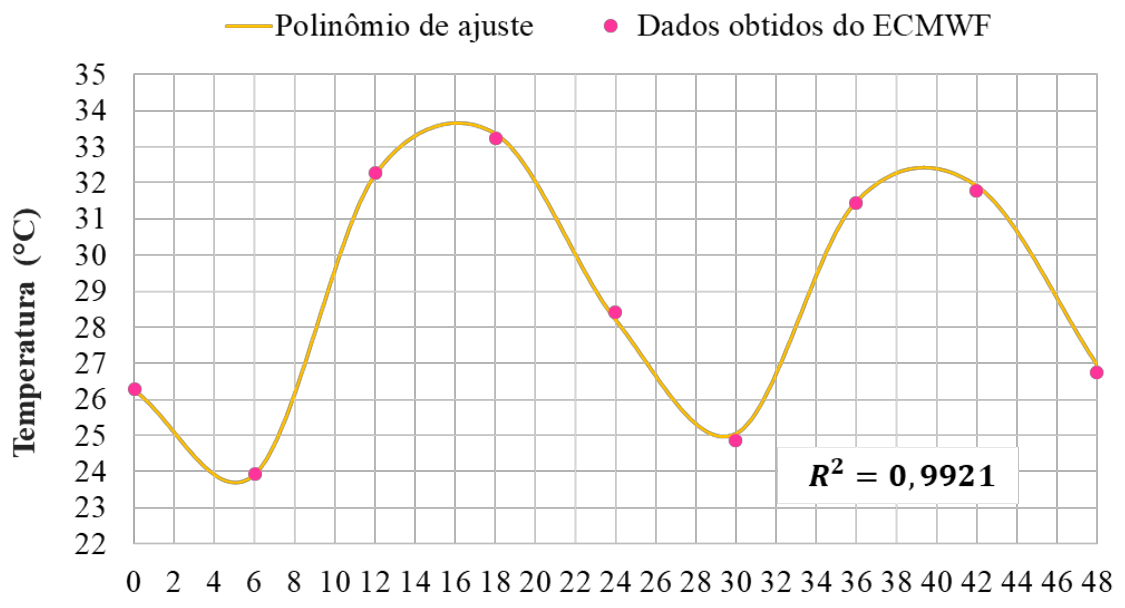

Tempo (h)

Fonte: European Centre for Medium-Range Weather Forecasts (2019). 


\section{Fundo}

$\mathrm{Na}$ fronteira inferior do domínio computacional foi considerada uma temperatura de $27,2^{\circ} \mathrm{C}$, que é a média aritmética da temperatura da camada $4(1 \mathrm{~m}-2,89 \mathrm{~m}$ de profundidade $)$ do solo de Belém, no mês de outubro de 2010, calculada usando as temperaturas em quatro momentos diferentes, $0 \mathrm{~h}, 6 \mathrm{~h}, 12 \mathrm{~h}$ e $18 \mathrm{~h}$, de cada dia desse mês, obtidas do site do ECMWF (EUROPEAN..., 2019). Assim, a fronteira inferior é definida como a condição de Dirichlet. Essa temperatura também foi utilizada como condição inicial para todo o modelo, na simulação transiente.

\section{Interface solo-ar}

Por simplicidade, a espessura da parede do tubo é negligenciada, devido à sua espessura relativamente pequena. Isso não afeta significativamente os resultados, pois é apontado por Brum et al. (2013), Ascione, Bellia e Minichiello (2011) e Bansal et al. (2010). Nessa interface é atribuída uma superfície antiderrapante para a condição de momento.

\section{Superfícies frontal, posterior e laterais}

As duas fronteiras laterais estão a $3 \mathrm{~m}$ do centro do tubo, isto é, mais de sete vezes o diâmetro do tubo. Portanto, esses limites são considerados adiabáticos como as superfícies frontal e posterior do modelo.

\section{Configuração da solução}

O algoritmo COUPLED é aplicado na solução transiente baseada em pressão. Um esquema upwind de segunda ordem é adotado para discretização espacial das equações governantes, e um esquema implícito de primeira ordem é escolhido para definir a formulação da solução dependente do tempo. $\mathrm{O}$ critério de convergência para energia é $10^{-6}$, enquanto para outros é $10^{-4}$. As simulações realizadas para este trabalho representam um intervalo de tempo de 2 dias. Assim, foi adotado um passo de tempo de $60 \mathrm{~s}$ com um total de 2.880 passos, e um máximo de 20 interações por passo de tempo.

\section{Teste de independência de malha}

Foram geradas sete malhas estruturadas com tamanhos de 16.236, 112.530, 219.114, 314.853, 411.511, 465.600 e 523.665 nós e o ponto central na saída do tubo é adotado para testar a independência da malha desse modelo. Para gerar essas malhas, volumes finitos hexaédricos foram utilizados. Os resultados da temperatura na saída do TCSA para diferentes tamanhos de malha são mostrados na Figura 8. A partir dessa figura, confirma-se que uma malha de 523.665 nós é precisa o suficiente para simular o TCSA nesta pesquisa. Assim, esse foi o número de nós da malha empregada para investigar numericamente o sistema TCSA.

Figura 8 - Temperatura do ar do ponto central na saída em diferentes malhas

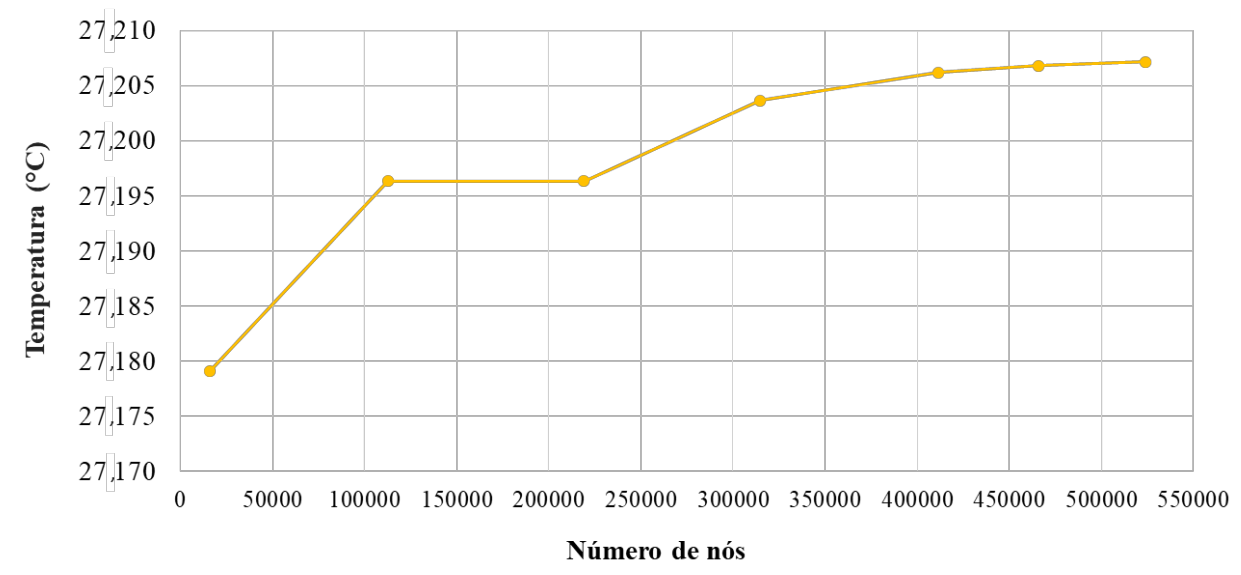


A estrutura da malha de 523.665 nós é mostrada na Figura 9. Como mostra o detalhe dessa figura, a malha é mais refinada na região interior do duto e perto da parede dele para aumentar precisão da simulação. Além disso, existem 80 camadas de malhas na direção $z$. O total de volumes criados para a discretização é de 502.080, dos quais 393.280 correspondem ao solo e 108.800 correspondem ao duto, que representa $21,7 \%$ do total de células, sendo o volume geométrico do duto de apenas $0,35 \%$ do volume do domínio completo. Após o processamento anteriormente, a qualidade dessa malha selecionada é 0,70928 na qualidade ortogonal mínima e 0,5 na assimetria máxima.

\section{Validação do modelo}

\section{Descrição da configuração experimental do TCSA}

Para a validação do modelo numérico desenvolvido nesta pesquisa, dados de temperatura de uma bancada de testes experimentais realizada pela equipe de N. Moummi na Universidade de Biskra $\left(34^{\circ} 47^{\prime} \mathrm{N}-5^{\circ} 43^{\prime} \mathrm{E}\right) \mathrm{em}$ 2009 (MOUMMI et al., 2010) foram usados. De acordo com Mehdid et al. (2018), a configuração experimental do TCSA é uma serpentina horizontal de tubo cilíndrico de PVC de $0.11 \mathrm{~m}$ de diâmetro interno e $47 \mathrm{~m}$ de comprimento. O TCSA é enterrado a uma profundidade de $3 \mathrm{~m}$ sob uma inclinação de $2 \%$ para a evacuação do ar condensado. As seções serpentinas horizontais do tubo são espaçadas a uma distância de cerca de $2 \mathrm{~m}$. A saída do tubo horizontal é conectada a um extrator de ar de fluxo variável através de um tubo vertical. Quatorze termopares foram usados para medir a temperatura do ar ao longo do tubo horizontal (T1, T2, T3, T4, T5, T6, T7, T8, T9, T10, T11, T12, T13, T14). Esses termopares são inseridos em diferentes posições ao longo do comprimento do tubo horizontal (Figura 10).

\section{Medições experimentais}

As experiências foram realizadas na Universidade Biskra no dia 2 de maio de 2013, das 9h45min às $15 \mathrm{~h} 45 \mathrm{~min}$. As temperaturas são medidas a cada 15 minutos para uma velocidade de fluxo de ar constante de $3,5 \mathrm{~m} / \mathrm{s}$. As medições experimentais das temperaturas do ar na saída do duto ao longo do dia obtidas a partir do par termoelétrico T14 (Figura 10) são apresentadas na Tabela 2 (MEHDID et al., 2018).

Figura 9 - Estrutura da malha de 523.665 nós - (a) seção transversal completa; (b) detalhe dos elementos do solo, mais próximo do duto; e (c) detalhe dos elementos dentro do tubo

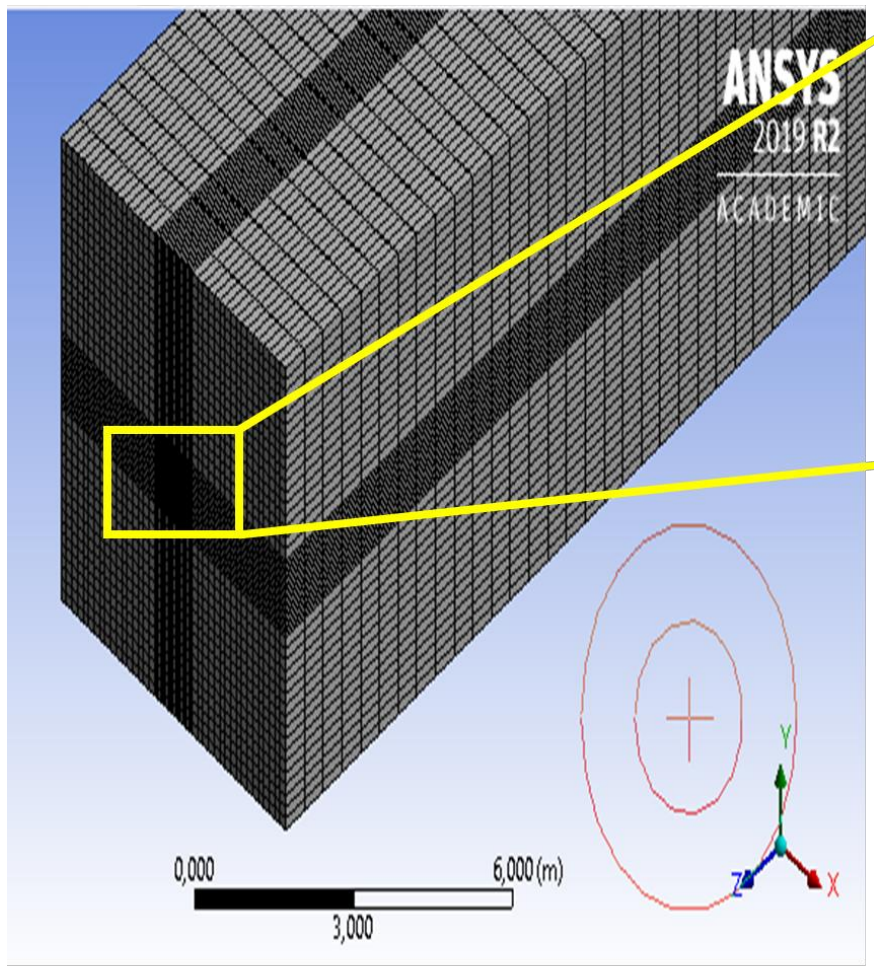

(a)

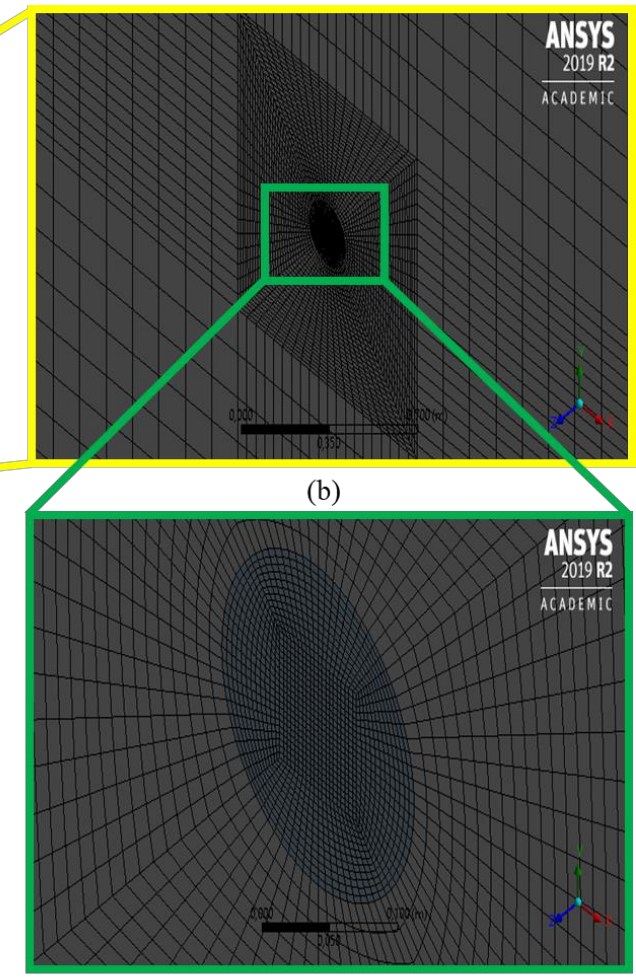

(c)

368 Vielma Vivas, G. A. V.; Guerra, D. R. S. 
Figura 10 - Posição dos termopares que medem as temperaturas do fluxo de ar (T1, T2, T3, T4, T5, T6, T7, T8, T9, T10, T11, T12, T13, T14) com as seguintes distâncias (da entrada TCSA): 0,00, 0,63, 4,69, $8,72,10,89,13,04,17,07,23,37,26,07,30,10,34,00,35,89,37,82,42,10,47,00 \mathrm{~m}$

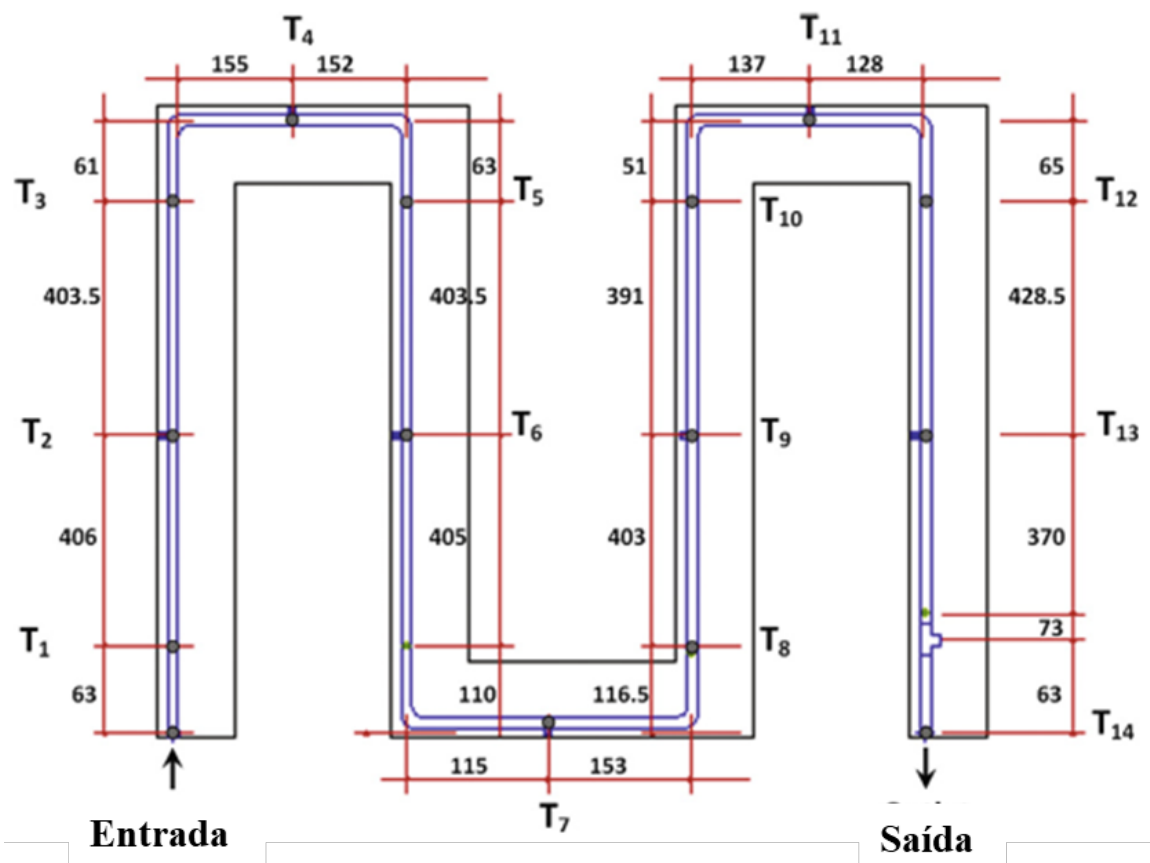

Fonte: adaptado de Mehdid et al. (2018).

Tabela 2 - Comparação das temperaturas do ar na saída do tubo obtidas a partir do modelo numérico com as medições experimentais realizadas em 2 de maio de 2013 na Universidade de Biskra (MEHDID et al., 2018)

\begin{tabular}{c|c|c|c|c|c}
\hline $\begin{array}{c}\text { Hora do } \\
\text { dia }\end{array}$ & $\begin{array}{c}\text { Tempo } \\
\text { (h) }\end{array}$ & $\begin{array}{c}\text { Temperatura do } \\
\text { ar na entrada do } \\
\text { duto }\left({ }^{\circ} \mathbf{C}\right)\end{array}$ & $\begin{array}{c}\text { Temperatura do ar } \\
\text { na saída do duto } \\
\text { experimental }\left({ }^{\circ} \mathbf{C}\right)\end{array}$ & $\begin{array}{c}\text { Temperatura do ar na } \\
\text { saída do duto } \\
\text { numérica }\left({ }^{\circ} \mathbf{C}\right)\end{array}$ & $\begin{array}{c}\text { Erro } \\
\text { relativo } \\
(\%)\end{array}$ \\
\hline 9h45min & 0,00 & 30,00 & 22,95 & 22,00 & 4,14 \\
$10 \mathrm{~h} 00 \mathrm{~min}$ & 0,25 & 29,50 & 22,98 & 22,08 & 3,93 \\
$10 \mathrm{~h} 15 \mathrm{~min}$ & 0,50 & 30,30 & 23,00 & 22,10 & 3,91 \\
$10 \mathrm{~h} 30 \mathrm{~min}$ & 0,75 & 31,30 & 23,02 & 22,12 & 3,90 \\
$10 \mathrm{~h} 45 \mathrm{~min}$ & 1,00 & 30,40 & 23,05 & 22,14 & 3,95 \\
$11 \mathrm{~h} 00 \mathrm{~min}$ & 1,25 & 31,40 & 23,06 & 22,16 & 3,91 \\
$11 \mathrm{~h} 15 \mathrm{~min}$ & 1,50 & 30,50 & 23,08 & 22,17 & 3,92 \\
$11 \mathrm{~h} 30 \mathrm{~min}$ & 1,75 & 31,60 & 23,09 & 22,19 & 3,90 \\
$11 \mathrm{~h} 45 \mathrm{~min}$ & 2,00 & 32,90 & 23,11 & 22,22 & 3,91 \\
$12 \mathrm{~h} 00 \mathrm{~min}$ & 2,25 & 32,50 & 23,13 & 22,24 & 3,93 \\
$12 \mathrm{~h} 15 \mathrm{~min}$ & 2,50 & 32,80 & 23,14 & 22,25 & 3,91 \\
$12 \mathrm{~h} 30 \mathrm{~min}$ & 2,75 & 32,60 & 23,15 & 22,26 & 3,89 \\
$12 \mathrm{~h} 45 \mathrm{~min}$ & 3,00 & 33,70 & 23,17 & 22,28 & 3,91 \\
$13 \mathrm{~h} 00 \mathrm{~min}$ & 3,25 & 34,40 & 23,17 & 22,29 & 3,85 \\
$13 \mathrm{~h} 15 \mathrm{~min}$ & 3,5 & 34,40 & 23,19 & 22,30 & 3,88 \\
$13 \mathrm{~h} 30 \mathrm{~min}$ & 3,75 & 33,70 & 23,21 & 22,32 & 3,90 \\
$13 \mathrm{~h} 45 \mathrm{~min}$ & 4,00 & 33,40 & 23,21 & 22,33 & 3,85 \\
$14 \mathrm{~h} 00 \mathrm{~min}$ & 4,25 & 32,40 & 23,22 & 22,34 & 3,84 \\
$14 \mathrm{~h} 15 \mathrm{~min}$ & 4,50 & 34,50 & 23,23 & 22,35 & 3,83 \\
$14 \mathrm{~h} 30 \mathrm{~min}$ & 4,75 & 34,00 & 23,24 & 22,36 & 3,82 \\
$14 \mathrm{~h} 45 \mathrm{~min}$ & 5,00 & 34,00 & 23,24 & 22,38 & 3,77 \\
$15 \mathrm{~h} 00 \mathrm{~min}$ & 5,25 & 34,30 & 23,26 & 22,39 & 3,80 \\
$15 \mathrm{~h} 15 \mathrm{~min}$ & 5,50 & 35,30 & 23,27 & 3,80 \\
$15 \mathrm{~h} 30 \mathrm{~min}$ & 5,75 & 35,00 & 23,28 & 3,79 \\
$15 \mathrm{~h} 45 \mathrm{~min}$ & 6,00 & 35,00 & 23,29 & 3,79 \\
\hline
\end{tabular}




\section{Validação experimental}

O modelo numérico desenvolvido foi validado com as medições de temperatura experimentais apresentadas nas Tabelas 2 e 4 . Os parâmetros físicos e térmicos dos diferentes materiais utilizados na validação estão listados na Tabela 3.

Por outro lado, uma temperatura de $22{ }^{\circ} \mathrm{C}$ foi usada como condição inicial de temperatura para todo $\mathrm{o}$ domínio computacional. Essa temperatura também foi considerada como condição de contorno para a superfície inferior do modelo no ANSYS Fluent. Em relação à entrada do duto, foi definida uma velocidade do ar de 3,5 m/s. Além disso, a partir dos dados de temperatura do ar ambiente apresentados na Tabela 2 , um polinômio de ajuste quadrático foi obtido usando o software R (THE R..., 2020) para usá-lo como condição de contorno da temperatura nessa entrada (Figura 11).

Com relação à condição de contorno na superfície superior do modelo, os dados de temperatura para os dias $1^{\circ}$ e 2 de maio de 2010 foram obtidos no site do ECMWF (EUROPEAN..., 2019). Como observado anteriormente, essa plataforma oferece apenas quatro temperaturas por dia, motivo pelo qual o autor decidiu considerar dois dias desse mês em vez de apenas 2 de maio e, portanto, possui 9 dados de temperatura para obter um melhor polinômio de ajuste. Nesse caso, 6 splines foram geradas para ajustar os dados através do software estadístico R (THE R..., 2020). Os valores de temperatura obtidos juntamente com as curvas de ajuste são mostrados na Figura 12.

Tabela 3 - Propriedades termofísicas (de ar e solo circundante) utilizadas na validação do modelo numérico desenvolvido

\begin{tabular}{c|c|c|c|c}
\hline Material & $\begin{array}{c}\text { Massa } \\
\text { específica } \\
\left(\mathbf{k g} / \mathbf{m}^{3}\right)\end{array}$ & $\begin{array}{c}\text { Calor } \\
\text { específico } \\
\left(\mathbf{J} /\left(\mathbf{k g}^{\circ} \mathbf{C}\right)\right)\end{array}$ & $\begin{array}{c}\text { Condutividade } \\
\text { térmica }\left(\mathbf{W} /\left(\mathbf{m}^{\circ} \mathbf{C}\right)\right)\end{array}$ & $\begin{array}{c}\text { Viscosidade } \\
\text { dinâmica } \\
(\mathbf{k g} /(\mathbf{m} \cdot \mathbf{s}))\end{array}$ \\
\hline $\mathrm{Ar}^{1}$ & 1,225 & 1005 & 0,0242 & $1,90 \times 10^{-5}$ \\
$\mathrm{Solo}^{1}$ & 1800 & 1340 & 1,5 & \\
\hline
\end{tabular}

Nota: ${ }^{1}$ as propriedades termofísicas do ar e do solo foram obtidas do trabalho de Mehdid et al. (2018).

Figura 11 - Dados experimentais da temperatura do ar na entrada do duto e o polinômio de ajuste correspondente

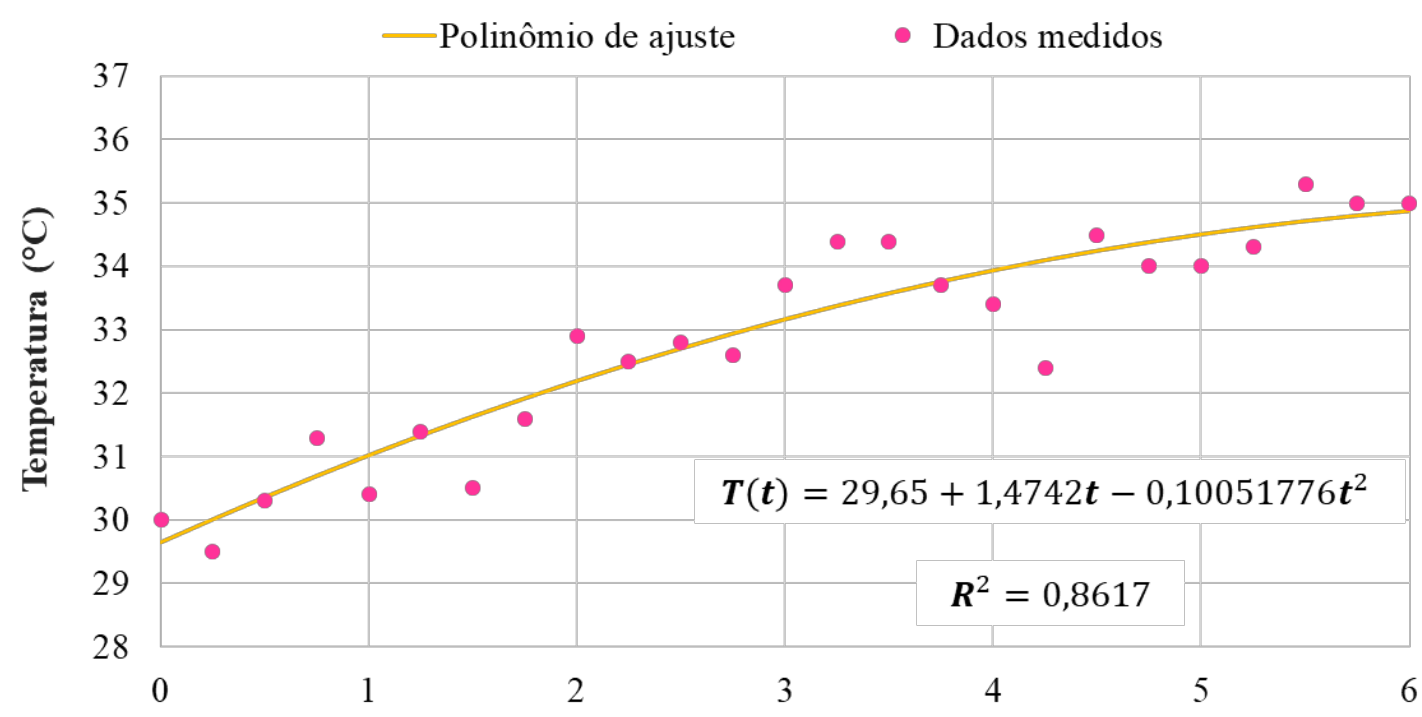

Tempo (h)

Fonte: Mehdid et al. (2018). 
Figura 12 - Dados de temperatura da superfície do solo obtidos do ECMWF e o polinômio de ajuste correspondente

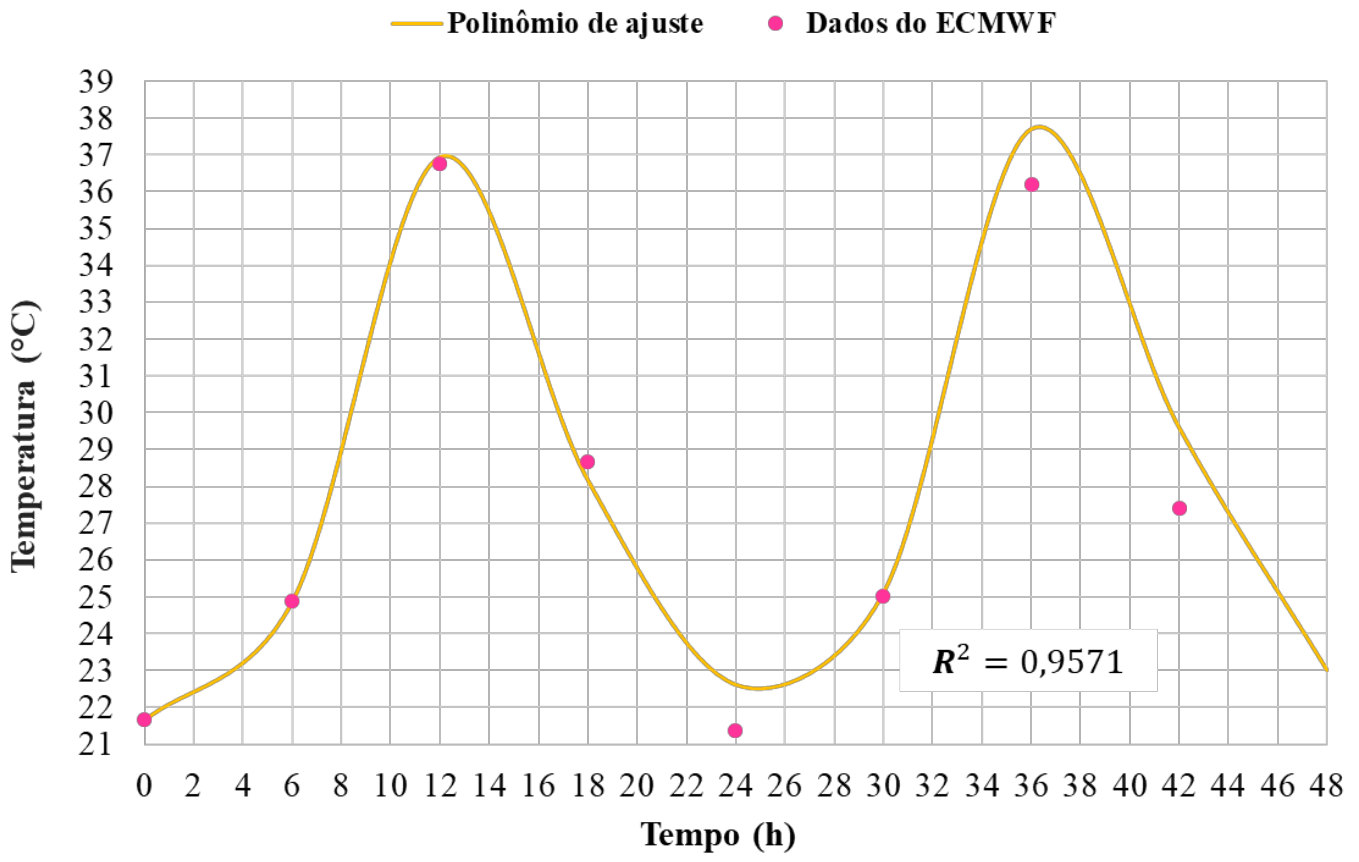

Fonte: European Centre for Medium-Range Weather Forecasts (2019).

Como a validação ocorre somente entre $9 \mathrm{~h} 45 \mathrm{~min}$ e $15 \mathrm{~h} 45 \mathrm{~min}$ de 2 de maio, somente as curvas entre $33,75 \mathrm{~h}$ e 39,75 h são de interesse. Essas curvas são dadas pela Equação 13:

$T(t)=\left\{\begin{array}{c}2423,928-215,992 t+6,425 t^{2}-0,063 t^{3}, \quad 33,75 \leq t<38 \\ -4506,284+331,130 t-7.973 t^{2}+0,063 t^{3}, \quad 38 \leq t \leq 39.75\end{array}\right.$

Eq. 13

Onde:

$t(h)$ é o tempo; e

$T\left({ }^{\circ} \mathrm{C}\right)$ é a temperatura.

A Equação 12 é então ajustada para que o tempo de início da simulação seja 33,75 h, ou seja, essas curvas são deslocadas 33,75 unidades para a esquerda, obtendo-se a Equação 14:

$T(t)= \begin{cases}32,429+2,550 t+0.050 t^{2}-0,063 t^{3}, & 0 \leq t<4.25 \\ 22,734+9,394 t-1.560 t^{2}+0,063 t^{3}, & 4.25 \leq t \leq 6\end{cases}$

A função de temperatura do ar na entrada da tubulação e a temperatura da superfície do solo são definidas no software ANSYS Fluent através de uma UDF.

A Tabela 2 mostra os valores de temperatura do ar na saída do tubo, obtidos a partir do modelo numérico e através de medições experimentais realizadas na Universidade Biskra. Verifica-se que existe uma boa concordância entre as temperaturas do ar previstas pelo presente modelo e as medições experimentais com um erro relativo máximo de 4,14\%. Além disso, a Tabela 4 e a Figura 13 mostram as temperaturas calculadas com o modelo numérico e os dados experimentais para $6 \mathrm{~h}$ de operação contínua em diferentes seções ao longo do TCSA. Nesse caso se tem um erro relativo máximo de 3,83\%.

\section{Resultados e discussões}

Em cada um dos casos mostrados a seguir, o comprimento do tubo é de $40 \mathrm{~m}$. 
Tabela 4 - Validação das temperaturas obtidas através do modelo numérico desenvolvido com resultados experimentais medidos após 6 horas de funcionamento ao longo do TCSA

\begin{tabular}{c|c|c|c}
\hline $\begin{array}{c}\text { Seção ao longo } \\
\text { do TCSA }(\mathbf{m})\end{array}$ & $\begin{array}{c}\text { Temperatura } \\
\text { experimental }\left({ }^{\circ} \mathbf{C}\right)\end{array}$ & $\begin{array}{c}\text { Temperatura } \\
\text { numérica }\left({ }^{\circ} \mathbf{C}\right)\end{array}$ & $\begin{array}{c}\text { Erro } \\
\text { relativo }(\%)\end{array}$ \\
\hline 0,00 & 35,00 & 34,88 & 0,35 \\
0,63 & 35,00 & 34,88 & 0,35 \\
4,69 & 31,60 & 32,15 & 1,74 \\
8,73 & 28,90 & 29,54 & 2,20 \\
10,89 & 27,80 & 28,41 & 2,21 \\
13,04 & 26,80 & 27,46 & 2,47 \\
17,07 & 25,50 & 26,03 & 2,09 \\
23,27 & 25,10 & 24,52 & 2,29 \\
25,97 & 24,70 & 24,06 & 2,61 \\
30,00 & 24,10 & 23,51 & 2,44 \\
33,91 & 23,90 & 23,12 & 3,26 \\
35,79 & 23,70 & 22,97 & 3,08 \\
37,72 & 23,60 & 22,84 & 3,24 \\
42,00 & 23,50 & 22,60 & 3,83 \\
47,00 & 23,20 & 22,41 & 3,42 \\
\hline
\end{tabular}

Figura 13 - Comparação das temperaturas obtidas a partir do modelo numérico desenvolvido com resultados experimentais medidos ao longo do TCSA após 6 horas de funcionamento

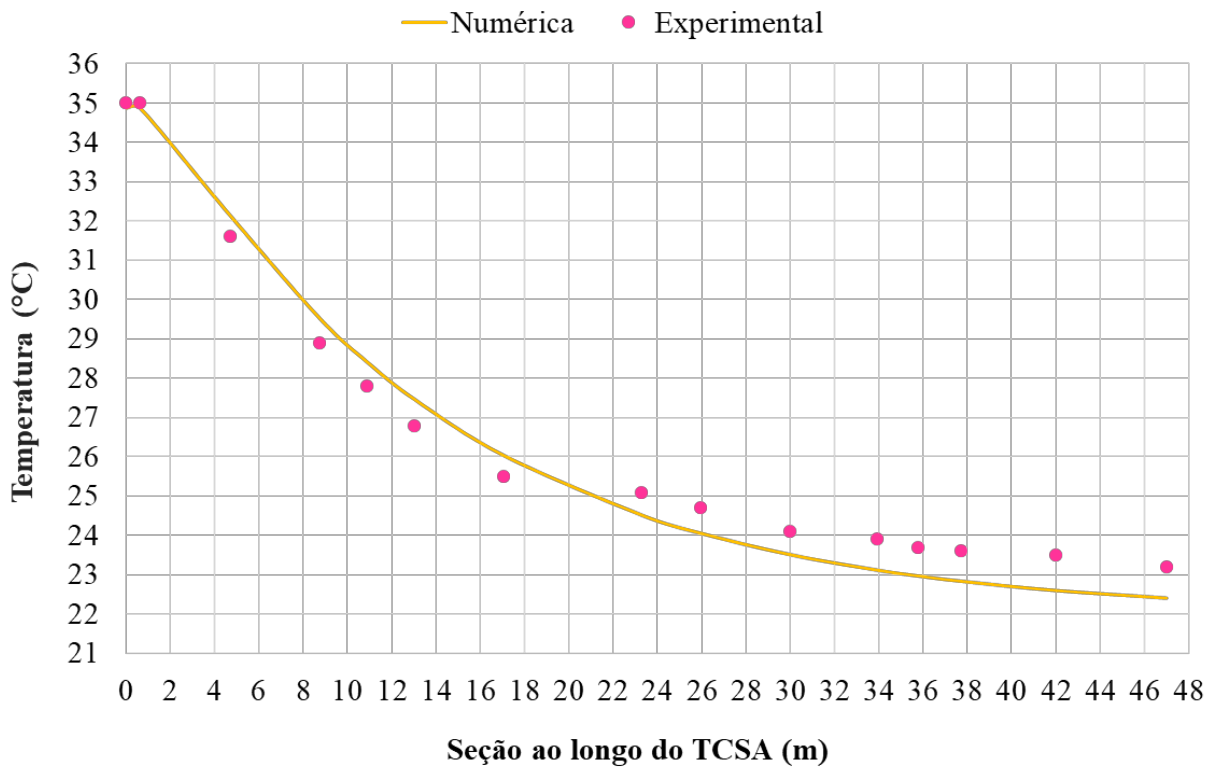

\section{Influência da velocidade do ar na queda de temperatura ao longo de diferentes seções do duto}

Caso 1: $v=0,5 \mathrm{~m} / \mathrm{s}, \mathrm{D}=0,2 \mathrm{~m}$

A Figura 14 mostra que durante o período de resfriamento entre aproximadamente $8 \mathrm{~h}$ e $20 \mathrm{~h}$, a temperatura do ar diminui à medida que escoa ao longo do comprimento do tubo. Por exemplo, às $14 \mathrm{~h} 40 \mathrm{~min}$, que é o tempo em que o ar atinge sua temperatura máxima na entrada, $33,15^{\circ} \mathrm{C}$, a temperatura do ar cai para 31,07 ${ }^{\circ} \mathrm{C}, 29,23{ }^{\circ} \mathrm{C}, 28,26{ }^{\circ} \mathrm{C}, 27,75^{\circ} \mathrm{C}, 27,49{ }^{\circ} \mathrm{C}, 27,35^{\circ} \mathrm{C}, 27,27{ }^{\circ} \mathrm{C}$ e $27,24{ }^{\circ} \mathrm{C}$, quando se desloca da entrada do tubo $5 \mathrm{~m}, 10 \mathrm{~m}, 15 \mathrm{~m}, 20 \mathrm{~m}, 25 \mathrm{~m}, 30 \mathrm{~m}, 35 \mathrm{~m}$ e $40 \mathrm{~m}$, respectivamente. Esses valores representam uma queda de temperatura de $2,08{ }^{\circ} \mathrm{C}, 3,92{ }^{\circ} \mathrm{C}, 4,89{ }^{\circ} \mathrm{C}, 5,39{ }^{\circ} \mathrm{C}, 5,66{ }^{\circ} \mathrm{C}, 5,80{ }^{\circ} \mathrm{C}, 5,87{ }^{\circ} \mathrm{C}$ e $5,91{ }^{\circ} \mathrm{C}$ para os comprimentos mencionados anteriormente. Ao comparar cada uma dessas quedas com a redução máxima de temperatura de $5,91{ }^{\circ} \mathrm{C}$, obtida a $40 \mathrm{~m}$ da entrada, as porcentagens de queda são de $35,21 \%, 66,27 \%$, $82,65 \%, 91,25 \%, 95,76 \%, 98,12 \%, 99,35 \%$, a $5 \mathrm{~m}, 10 \mathrm{~m}, 15 \mathrm{~m}, 20 \mathrm{~m}, 25 \mathrm{~m}, 30 \mathrm{~m}$ e $35 \mathrm{~m}$ a partir da entrada

372 Vielma Vivas, G. A. V.; Guerra, D. R. S. 
do tubo, respectivamente. Esses resultados mostram que, para o caso considerado, uma queda de temperatura superior a $90 \%$ da redução total ao longo de todo o comprimento do trocador de calor é alcançada a $20 \mathrm{~m}$ da entrada. O comprimento do tubo, no qual $90 \%$ da queda total da temperatura do ar é obtida, é definido como o ponto do joelho, e é um parâmetro usado para definir o desempenho térmico do TCSA (MISRA et al., 2013c; AGRAWAL et al., 2018).

\section{Caso 2: $\mathrm{v}=1,25 \mathrm{~m} / \mathrm{s}, \mathrm{D}=0,2 \mathrm{~m}$}

Na Figura 15 pode ser visto que, como no caso 1, durante o período de resfriamento, a temperatura do ar também diminui à medida que escoa ao longo do comprimento do tubo. Pelo mesmo tempo considerado no caso 1 , ou seja, às $14 \mathrm{~h} 40 \mathrm{~min}$, a temperatura do ar cai de $33,15^{\circ} \mathrm{C}$ na entrada, para $32,37{ }^{\circ} \mathrm{C}, 30,83{ }^{\circ} \mathrm{C}, 29,82$ ${ }^{\circ} \mathrm{C}, 29,09{ }^{\circ} \mathrm{C}, 28,56{ }^{\circ} \mathrm{C}, 28,18^{\circ} \mathrm{C}, 27,91{ }^{\circ} \mathrm{C}$ e $27,71{ }^{\circ} \mathrm{C}$, quando se desloca da entrada do tubo, $5 \mathrm{~m}, 10 \mathrm{~m}, 15$ $\mathrm{m}, 20 \mathrm{~m}, 25 \mathrm{~m}, 30 \mathrm{~m}, 35 \mathrm{~m}$ e $40 \mathrm{~m}$, respectivamente. Esses valores representam uma queda de temperatura de $0,78{ }^{\circ} \mathrm{C}, 2,32{ }^{\circ} \mathrm{C}, 3,33{ }^{\circ} \mathrm{C}, 4,06{ }^{\circ} \mathrm{C}, 4,58{ }^{\circ} \mathrm{C}, 4,97{ }^{\circ} \mathrm{C}, 5,24{ }^{\circ} \mathrm{C}$ e $5,44{ }^{\circ} \mathrm{C}$ para os comprimentos mencionados anteriormente. Ao comparar novamente, cada uma dessas quedas com a redução máxima de temperatura de $5,44{ }^{\circ} \mathrm{C}$, obtida a $40 \mathrm{~m}$ da entrada, as porcentagens de queda são de $14,38 \%, 42,65 \%$, $61,13 \%, 74,55 \%, 84,25 \%, 91,27 \%, 96,33 \%$, a $5 \mathrm{~m}, 10 \mathrm{~m}, 15 \mathrm{~m}, 20 \mathrm{~m}, 25 \mathrm{~m}, 30 \mathrm{~m}, 35 \mathrm{~m}$ a partir da entrada do tubo, respectivamente. Esses resultados mostram que nesse caso uma queda de temperatura superior a $90 \%$ da redução total ao longo de todo o comprimento do trocador do calor é atingida a $30 \mathrm{~m}$ da entrada. Observa-se que, diferentemente do caso 1, em que ocorreu a maior parte da queda de temperatura nos primeiros $20 \mathrm{~m}$ do tubo, nesse caso essa redução é realizada de maneira mais progressiva. Assim, é necessário que o ar viaje $30 \mathrm{~m}$ dentro do tubo para alcançar o ponto do joelho.

Figura 14 - Temperatura do ar no centro do duto $(v=0,5 \mathrm{~m} / \mathrm{s}, L=40 \mathrm{~m}$ e $D=0,2 \mathrm{~m})$

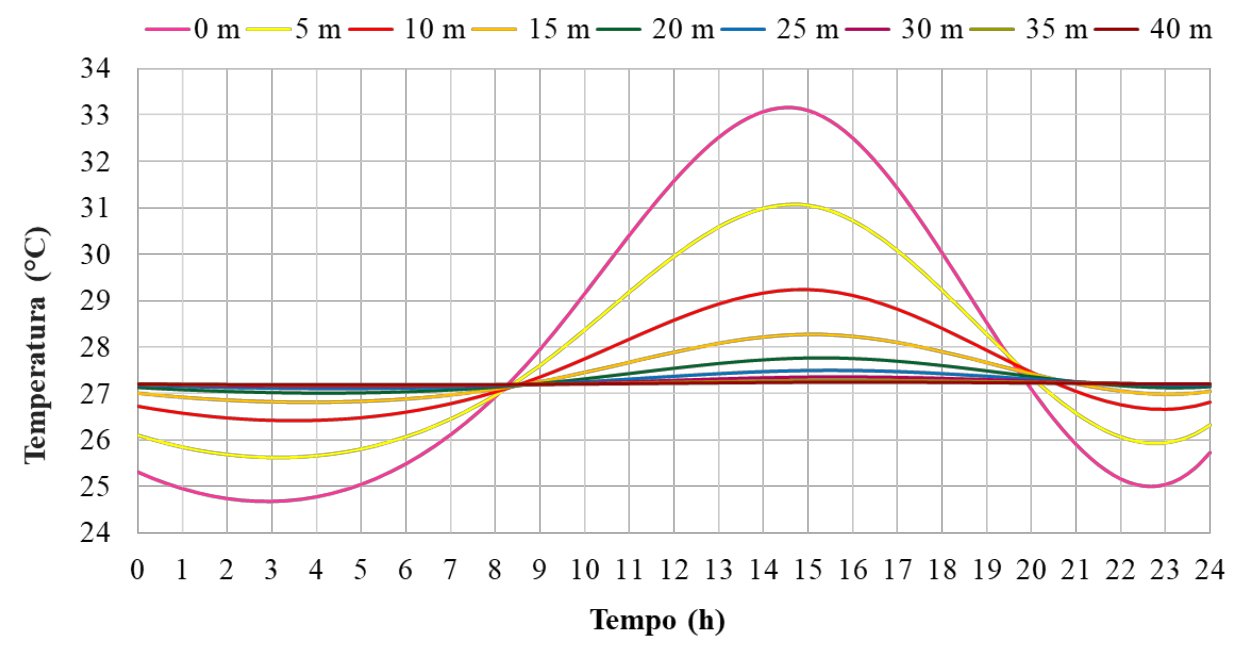

Figura 15 - Temperatura do ar no centro do duto $(v=1,25 \mathrm{~m} / \mathrm{s}, L=40 \mathrm{~m}$ e $D=0,2 \mathrm{~m})$

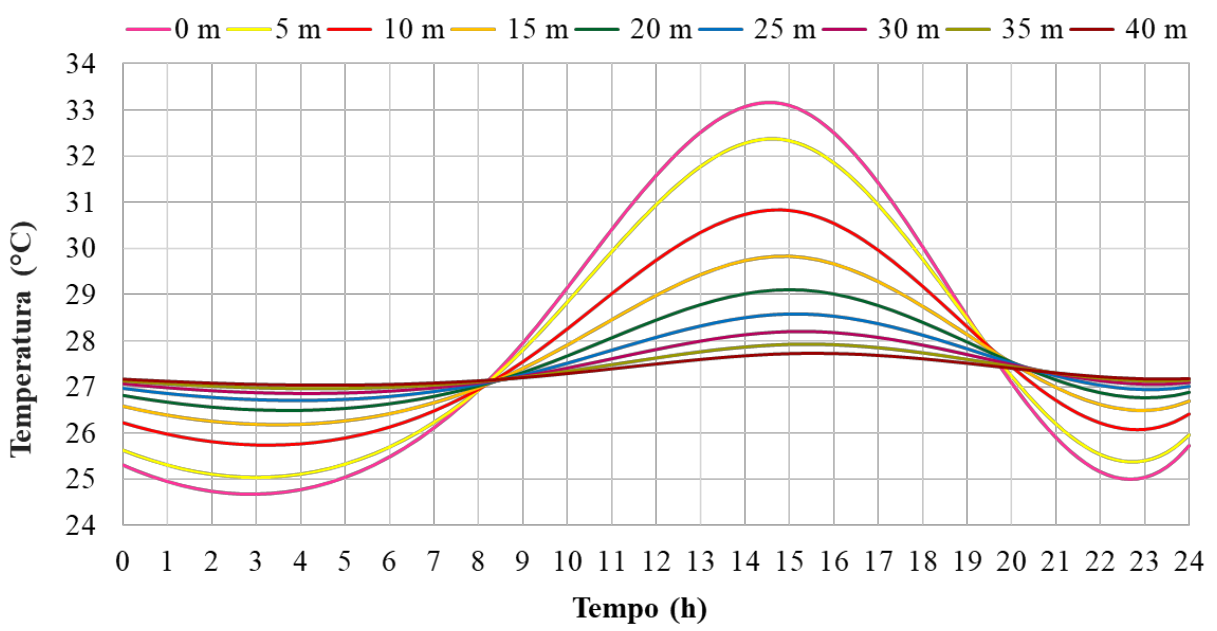




\section{Caso 3: $v=2 \mathrm{~m} / \mathrm{s}, \mathrm{D}=0,2 \mathrm{~m}$}

Nesse caso, o comportamento é semelhante aos dois casos anteriores, no que diz respeito à redução da temperatura ao longo da tubulação (Figura 16). Pelo mesmo tempo considerado nos casos 1 e 2 , a temperatura do ar cai de $33.15^{\circ} \mathrm{C}$ na entrada para $32,72{ }^{\circ} \mathrm{C}, 31,34{ }^{\circ} \mathrm{C}, 30,44{ }^{\circ} \mathrm{C}, 29,73{ }^{\circ} \mathrm{C}, 29,17{ }^{\circ} \mathrm{C}, 28,74$ ${ }^{\circ} \mathrm{C}, 28,40{ }^{\circ} \mathrm{C}$ e $28,13{ }^{\circ} \mathrm{C}$, quando se desloca da entrada do tubo $5 \mathrm{~m}, 10 \mathrm{~m}, 15 \mathrm{~m}, 20 \mathrm{~m}, 25 \mathrm{~m}, 30 \mathrm{~m}, 35 \mathrm{~m}$ e $40 \mathrm{~m}$, respectivamente. Esses valores representam uma queda de temperatura de $0,43{ }^{\circ} \mathrm{C}, 1,80{ }^{\circ} \mathrm{C}, 2,71{ }^{\circ} \mathrm{C}$, $3,42{ }^{\circ} \mathrm{C}, 3,97{ }^{\circ} \mathrm{C}, 4,41{ }^{\circ} \mathrm{C}, 4,75{ }^{\circ} \mathrm{C}$ e $5,02{ }^{\circ} \mathrm{C}$ para os comprimentos mencionados anteriormente. Ao comparar novamente cada uma dessas quedas com a redução máxima de temperatura de $5,02{ }^{\circ} \mathrm{C}$, obtida a 40 $\mathrm{m}$ da entrada, as porcentagens de queda são de $8,57 \%, 36,02 \%, 53,94 \%, 68,12 \%, 79,21 \%, 87,89 \%, 94,68 \%$, a $5 \mathrm{~m}, 10 \mathrm{~m}, 15 \mathrm{~m}, 20 \mathrm{~m}, 25 \mathrm{~m}, 30 \mathrm{~m}, 35 \mathrm{~m}$ a partir da entrada do tubo, respectivamente. Esses resultados permitem concluir que uma queda de temperatura superior a $90 \%$ da redução total ao longo de todo o comprimento do trocador do calor agora é alcançada a $35 \mathrm{~m}$ da entrada.

Os resultados obtidos nos três casos considerados demonstram que a velocidade do ar tem uma influência importante na distância necessária a partir da entrada do tubo, de modo que $90 \%$ da queda de temperatura obtida para o comprimento total do trocador de calor seja atingida. A diminuição da velocidade reduz esse parâmetro. A razão para isso pode ser que, à baixa velocidade, o ar permanece por mais tempo dentro do duto, aumentando a transferência de calor com o solo circundante, o que permite que desça sua temperatura a uma distância menor da entrada da tubulação. Também é importante observar que a necessidade de tubos longos para atingir certa queda de temperatura diminui reduzindo a velocidade do ar. Esse é o caso quando a velocidade do ar é de $2 \mathrm{~m} / \mathrm{s}$, em que são necessários $40 \mathrm{~m}$ de tubo para atingir uma queda de temperatura máxima de $5,02{ }^{\circ} \mathrm{C}$, enquanto uma redução de $5,399{ }^{\circ} \mathrm{C}$ é alcançada a $20 \mathrm{~m}$ da entrada do duto quando a velocidade do ar é de $0,5 \mathrm{~m} / \mathrm{s}$. Esses resultados são muito importantes, considerando uma das limitações práticas para a aplicação do TCSA em larga escala, ou seja, as grandes demandas de ocupação do solo durante a construção, particularmente em áreas densamente construídas (SONI; PANDEY; BARTARIA, 2016; LIU et al., 2019).

\section{Influência da velocidade do ar na entrada do duto no potencial térmico e no calor transferido pelo TCSA}

Brum et al. (2016b) definem o potencial térmico instantâneo do TCSA como uma média das diferenças de temperatura entre a saída e a entrada dos dutos. Devido às condições de temperatura da cidade de Belém, este estudo está interessado apenas no potencial de resfriamento do TCSA. Portanto, o potencial térmico foi redefinido para ser positivo quando o trocador estiver operando nessa condição. Assim, nesta pesquisa, em que o trocador tem apenas um duto, esse potencial é dado pela Equação 15:

$P(t)=T_{e}(t)-T_{S}(t)$

Onde $T_{e}(t)$ e $T_{s}(t)$ são, respectivamente, as temperaturas na entrada e na saída do duto no momento $t$.

Figura 16 - Temperatura do ar no centro do duto $(v=2 \mathrm{~m} / \mathrm{s}, L=40 \mathrm{~m}$ e $D=0,2 \mathrm{~m})$

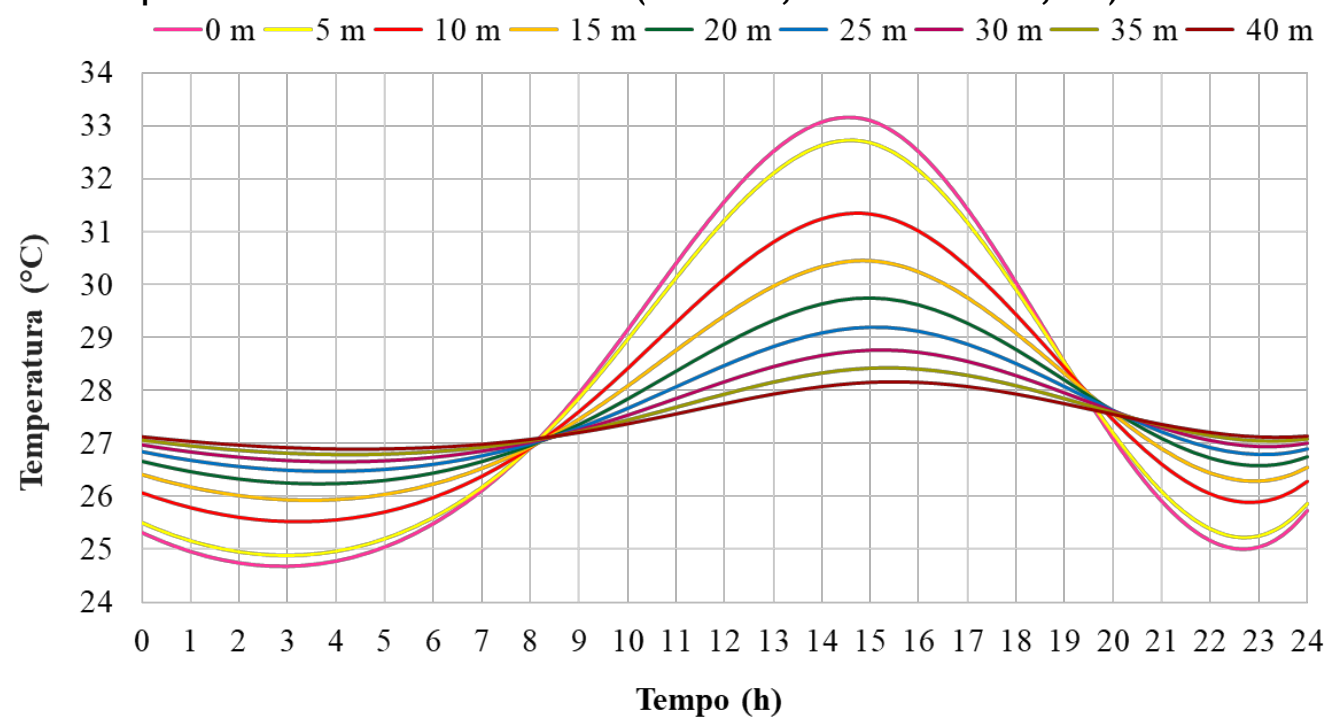

374 Vielma Vivas, G. A. V.; Guerra, D. R. S. 
As Figuras 17, 18 e 19 mostram o potencial térmico instantâneo para um diâmetro de tubo de 0,2 m, 0,3 m e $0,4 \mathrm{~m}$, respectivamente, considerando as velocidades de $0,5 \mathrm{~m} / \mathrm{s}, 1,25 \mathrm{~m} / \mathrm{s}$ e $2 \mathrm{~m} / \mathrm{s}$ para cada diâmetro. Nessas figuras, pode-se observar que para cada um dos diâmetros considerados esse potencial é aumentado pela redução da velocidade do ar na entrada da tubulação. Esse comportamento foi observado em outras investigações realizadas anteriormente (BRUM et al., 2016b; AGRAWAL; MISRA; AGRAWAL, 2020). A justificativa para isso pode ser que, ao diminuir a velocidade do ar, ele permanece por mais tempo dentro da tubulação, aumentando a transferência de calor com o solo circundante, diminuindo a temperatura de saída e, consequentemente, o potencial térmico instantâneo. A Tabela 5 mostra o potencial térmico instantâneo máximo para cada um dos diâmetros e velocidades considerados neste estudo. Os valores máximos alcançados às $14 \mathrm{~h} 40 \mathrm{~min}$ foram de $5,91{ }^{\circ} \mathrm{C}, 5,58{ }^{\circ} \mathrm{C}$ e $4,98{ }^{\circ} \mathrm{C}$, para diâmetros de $0,2 \mathrm{~m}, 0,3 \mathrm{~m} \mathrm{e} 0,4 \mathrm{~m}$, respectivamente, quando o ar escoa a $0,5 \mathrm{~m} / \mathrm{s}$.

Igualmente, a partir dos resultados da Tabela 5 , conclui-se que, à medida que a velocidade do ar aumenta de $0,5 \mathrm{~m} / \mathrm{s}$ para $1,25 \mathrm{~m} / \mathrm{s}$ e de $0,5 \mathrm{~m} / \mathrm{s}$ para $2 \mathrm{~m} / \mathrm{s}$, o potencial térmico instantâneo máximo é reduzido em 7,78\% e $14,89 \%, 19,53 \%$ e $29,21 \%$, e $27,11 \%$ e $37,75 \%$, para diâmetros de $0,2 \mathrm{~m}, 0,3 \mathrm{~m}$ e $0,4 \mathrm{~m}$, respectivamente. Esses resultados demonstram que essa redução é acelerada pelo aumento do diâmetro do duto.

Por outro lado, de acordo com Brum et al. (2016b) o potencial térmico $P_{t}$ é uma média mensal do potencial térmico instantâneo. Nesse caso, dado um mês entre os dias $t_{o}$ e $t_{n}$, as médias podem ser calculadas através da Equação 16:

$P_{t}=\frac{1}{t_{n}-t_{0}} \int_{t_{0}}^{t_{n}} P(t) d t$

Eq. 16

Figura 17 - Potencial térmico instantâneo para três velocidades diferentes $(L=40 \mathrm{~m}$ e $D=0,2 \mathrm{~m})$

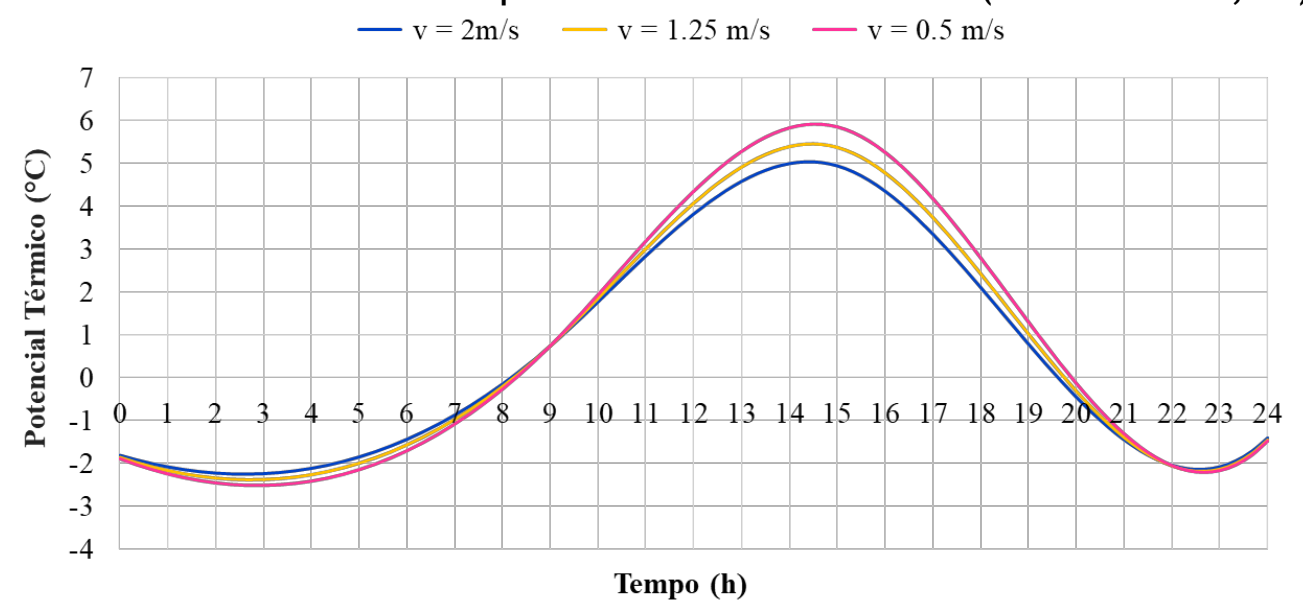

Figura 18 - Potencial térmico instantâneo para três velocidades diferentes $(L=40 \mathrm{~m}$ e $D=0,3 \mathrm{~m})$

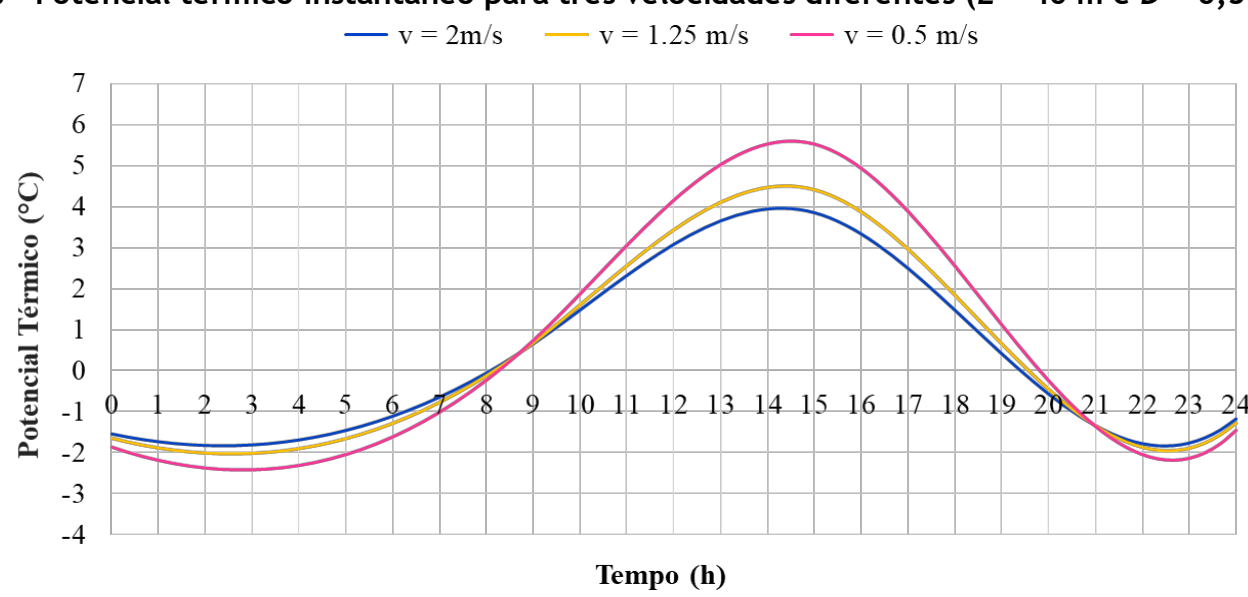


Figura 19 - Potencial térmico instantâneo para três velocidades diferentes $(L=40 \mathrm{~m}$ e $D=0,4 \mathrm{~m})$

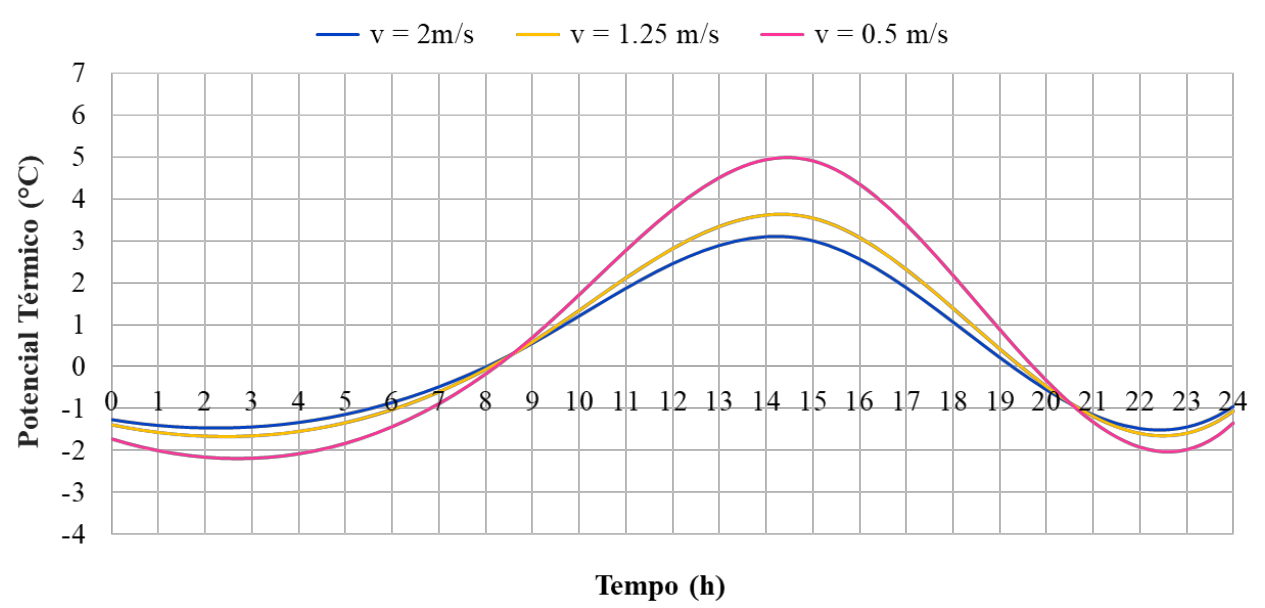

Esse potencial é muito importante, uma vez que o desempenho energético e a eficiência do TCSA são diretamente proporcionais ao $P_{t}$. Como nessa investigação as análises são feitas considerando apenas o segundo dia da simulação, ou seja, $1^{\circ}$ de outubro de 2010, a Equação 16 foi usada para calcular o potencial térmico médio diário de resfriamento. Para definir a função a ser usada na integral dessa equação, os polinômios de interpolação foram determinados usando spline quadrático para cada caso, com base nos valores de potencial térmico instantâneos obtidos na simulação. A Tabela 5 mostra os valores desse potencial médio para os diferentes casos estudados. Os valores máximos foram de $3,59{ }^{\circ} \mathrm{C}, 3,38{ }^{\circ} \mathrm{C}$ e 3,08 ${ }^{\circ} \mathrm{C}$, para diâmetros de $0,2 \mathrm{~m}, 0,3 \mathrm{~m}$ e $0,4 \mathrm{~m}$, respectivamente, quando o ar escoa a $0,5 \mathrm{~m} / \mathrm{s}$.

Em relação ao potencial térmico médio diário de resfriamento, novamente usando a Tabela 5, é determinado que à medida que a velocidade do ar aumenta de $0,5 \mathrm{~m} / \mathrm{s}$ para $1,25 \mathrm{~m} / \mathrm{s}$ e de $0,5 \mathrm{~m} / \mathrm{s} \mathrm{para} 2 \mathrm{~m} / \mathrm{s}$, esse potencial é reduzido em $8,36 \%$ e $13,37 \%, 18,05 \%$ e $28,40 \%$, e $27,60 \%$ e $37,01 \%$, para diâmetros de $0,2 \mathrm{~m}$, $0,3 \mathrm{~m}$ e $0,4 \mathrm{~m}$, respectivamente.

Para calcular o desempenho energético diário $Q$ do TCSA, pode-se usar a Equação 17 (INCROPERA et al., 2007; BRUM et al., 2016b):

$Q=\rho_{a} c_{a} P_{t} v \frac{\pi D^{2}}{4}$

Onde os valores para a massa específica $\rho_{a}$ e calor específico do ar $c_{a}$ são apresentados na Tabela 1 . Os valores de desempenho energético diário, considerando apenas as horas do dia em que o TCSA produz resfriamento, são mostrados na Tabela 5. Os valores mostrados permitem estabelecer que, embora o aumento na velocidade do ar no duto diminua o potencial médio, ele também aumenta $Q$. Os valores máximos foram de $228,78 \mathrm{~W}, 400,56 \mathrm{~W}$ e $570,86 \mathrm{~W}$, para diâmetros de $0,2 \mathrm{~m}, 0,3 \mathrm{~m}$ e $0,4 \mathrm{~m}$, respectivamente, quando o ar escoa a $2 \mathrm{~m} / \mathrm{s}$. A razão para esse comportamento está relacionada ao fato de que, embora o desempenho energético seja proporcional ao potencial térmico médio e à velocidade do ar, a porcentagem de queda desse potencial é excedida pelo aumento da velocidade. É o caso, por exemplo, da queda de $37,01 \%$ no potencial médio quando a velocidade aumenta de $0,5 \mathrm{~m} / \mathrm{s}$ para $2 \mathrm{~m} / \mathrm{s}$, o que corresponde a um aumento de velocidade de $300 \%$.

De acordo com a Tabela 5 , à medida que a velocidade do ar aumenta de $0,5 \mathrm{~m} / \mathrm{s}$ para $1,25 \mathrm{~m} / \mathrm{s}$ e de $0,5 \mathrm{~m} / \mathrm{s}$ para $2 \mathrm{~m} / \mathrm{s}$, o desempenho energético diário também aumenta em 152,02\% e 281,17\%, 104,88\% e 186,40\%, e $81 \%$ e $151,95 \%$, para diâmetros de $0,2 \mathrm{~m}, 0,3 \mathrm{~m}$ e $0,4 \mathrm{~m}$, respectivamente. Esses resultados demonstram que esse aumento é reduzido à medida que o diâmetro do duto aumenta.

\section{Influência do diâmetro do duto no potencial térmico e no calor transferido pelo TCSA}

As Figuras 20, 21 e 22 mostram o potencial térmico instantâneo para as velocidades de $0,5 \mathrm{~m} / \mathrm{s}, 1,25 \mathrm{~m} / \mathrm{s}$ e 2 $\mathrm{m} / \mathrm{s}$, respectivamente, considerando diâmetros de tubo de $0,2 \mathrm{~m}, 0,3 \mathrm{~m}$ e $0,4 \mathrm{~m}$, para cada velocidade. Nessas três figuras pode-se observar que para cada uma das velocidades consideradas esse potencial é aumentado pela redução do diâmetro do duto. De acordo com a Tabela 5 , os valores máximos de $5,91{ }^{\circ} \mathrm{C}, 5,45{ }^{\circ} \mathrm{C}$ e 5,03

376 Vielma Vivas, G. A. V.; Guerra, D. R. S. 
${ }^{\circ} \mathrm{C}$ foram atingidos às $14 \mathrm{~h} 40 \mathrm{~min}$, para velocidades de $0,5 \mathrm{~m} / \mathrm{s}, 1,25 \mathrm{~m} / \mathrm{s}$ e $2 \mathrm{~m} / \mathrm{s}$, respectivamente, quando o diâmetro de duto é de 0,2 m. Esse comportamento foi relatado anteriormente nos trabalhos de Brum et al. (2016b) e Agrawal, Misra e Agrawal (2020). A razão desses resultados pode ser atribuída ao fato de que a distância entre o centro do tubo e a camada do solo diminui com a redução do diâmetro do tubo, o que pode facilitar a transferência de calor das camadas de ar próximas do centro do duto para o solo circundante.

Usando os resultados da Tabela 5 , pode-se concluir que à medida que o diâmetro do duto aumenta de $0,2 \mathrm{~m}$ para $0,3 \mathrm{~m}$ e de $0,2 \mathrm{~m}$ para $0,4 \mathrm{~m}$ o potencial térmico instantâneo máximo diminui em 5,58\% e $15,74 \%$, $17,61 \%$ e $33,33 \%$, e $21,47 \%$ e $38,37 \%$, para velocidades de $0,5 \mathrm{~m} / \mathrm{s}, 1,25 \mathrm{~m} / \mathrm{s}$ e $2 \mathrm{~m} / \mathrm{s}$, respectivamente. Esses resultados demonstram que essa redução é acelerada pelo aumento da velocidade do ar.

Em relação ao potencial térmico médio diário de resfriamento, nesse caso os valores máximos são $3,59{ }^{\circ} \mathrm{C}$, $3,29{ }^{\circ} \mathrm{C}$ e $3,11^{\circ} \mathrm{C}$, para velocidades de $0,5 \mathrm{~m} / \mathrm{s}, 1,25 \mathrm{~m} / \mathrm{s}$ e $2 \mathrm{~m} / \mathrm{s}$, respectivamente, também obtidos com um diâmetro de $0,2 \mathrm{~m}$. Novamente usando a Tabela 5 , é determinado que à medida que o diâmetro do duto aumenta de $0,2 \mathrm{~m}$ para $0,3 \mathrm{~m}$ e de $0,2 \mathrm{~m}$ para $0,4 \mathrm{~m}$ esse potencial diminui em $5,85 \%$ e $14,21 \%, 15,81 \%$ e $32,22 \%$, e $22,19 \%$ e $37,62 \%$, para velocidades de $0,5 \mathrm{~m} / \mathrm{s}, 1,25 \mathrm{~m} / \mathrm{s}$ e $2 \mathrm{~m} / \mathrm{s}$, respectivamente.

Além disso, de acordo com a Tabela 5 , em relação ao desempenho energético diário, os valores máximos foram de $226,58 \mathrm{~W}, 410,12 \mathrm{~W}$ e $570,86 \mathrm{~W}$, para velocidades de $0,5 \mathrm{~m} / \mathrm{s}, 1,25 \mathrm{~m} / \mathrm{s}$ e $2 \mathrm{~m} / \mathrm{s}$, respectivamente, obtidos com um diâmetro de $0,4 \mathrm{~m}$. Como no caso da velocidade, apontando anteriormente, embora o aumento no diâmetro diminua o potencial médio, ele também aumenta $Q$. A razão para esse comportamento está relacionada ao fato de que, embora o desempenho energético seja proporcional ao potencial térmico médio, ele também é função do quadrado do diâmetro do duto, portanto a porcentagem de queda desse potencial é excedida pelo aumento do diâmetro. É o caso, por exemplo, da queda de 37,62\% no potencial médio quando o diâmetro aumenta de $0,2 \mathrm{~m}$ para $0,4 \mathrm{~m}$, o que implica que o quadrado do diâmetro varia de 0,04 a 0,16 , ou seja, um aumento de $300 \%$.

Tabela 5 - Potencial térmico instantâneo máximo, potencial térmico de resfriamento médio e desempenho energético diário para três diferentes diâmetros

\begin{tabular}{c|c|c|c|c|c}
\hline $\boldsymbol{D}(\mathbf{m})$ & $\boldsymbol{v}(\mathbf{m} / \mathbf{s})$ & $\begin{array}{c}\text { Número de } \\
\text { Reynolds }\end{array}$ & $\begin{array}{c}\text { Potencial térmico } \\
\text { instantâneo } \\
\text { máximo }\left({ }^{\circ} \mathbf{C}\right)\end{array}$ & $\begin{array}{c}\text { Potencial térmico } \\
\text { de resfriamento } \\
\text { médio }\left({ }^{\circ} \mathbf{C}\right)\end{array}$ & $\boldsymbol{Q}(\mathbf{W})$ \\
\hline \multirow{3}{*}{0,20} & 0,50 & 6131,57 & 5,91 & 3,59 & 60,02 \\
& 1,25 & 15328,94 & 5,45 & 3,29 & 151,26 \\
& 2,00 & 24526,31 & 5,03 & 3,11 & 228,78 \\
\hline \multirow{3}{*}{0,30} & 0,50 & 9197,36 & 5,58 & 3,38 & 139,86 \\
& 1,25 & 22993,42 & 4,49 & 2,77 & 286,55 \\
& 2,00 & 36789,47 & 3,95 & 2,42 & 400,56 \\
\hline \multirow{3}{*}{0,40} & 0,50 & 12263,15 & 4,98 & 3,08 & 226,58 \\
& 1,25 & 30657,89 & 3,63 & 2,23 & 410,12 \\
& 2,00 & 49052,63 & 3,10 & 1,94 & 570,86 \\
\hline
\end{tabular}

Figura 20 - Potencial térmico instantâneo para três diâmetros diferentes $(L=40 \mathrm{~m} \mathrm{e} v=0,5 \mathrm{~m} / \mathrm{s})$

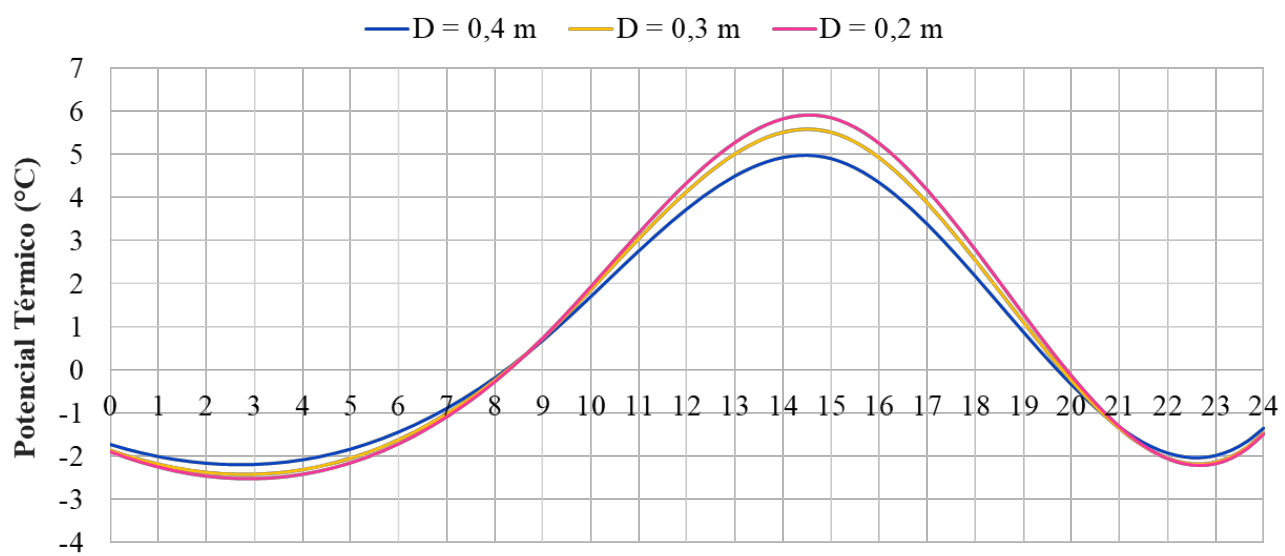

Tempo (h) 
Figura 21 - Potencial térmico instantâneo para três diâmetros diferentes $(L=40 \mathrm{~m} \mathrm{e} v=1,25 \mathrm{~m} / \mathrm{s})$

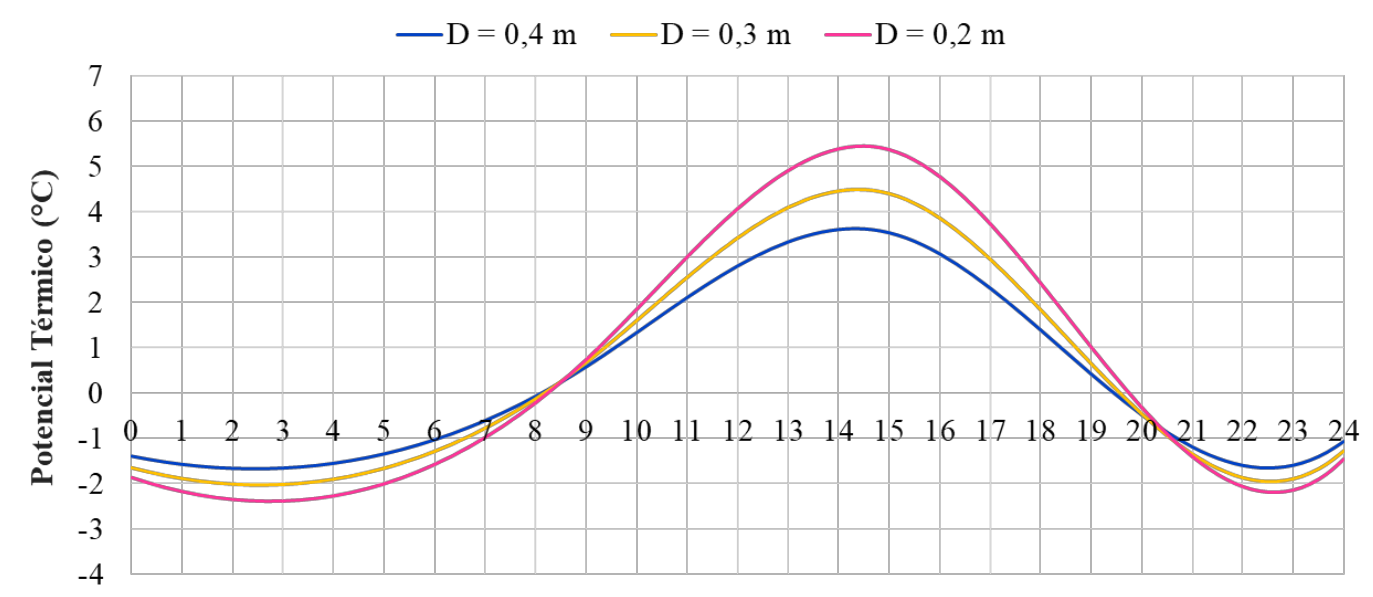

Tempo (h)

Figura 22 - Potencial térmico instantâneo para três diâmetros diferentes $(L=40 \mathrm{~m} \mathrm{e} v=2 \mathrm{~m} / \mathrm{s})$

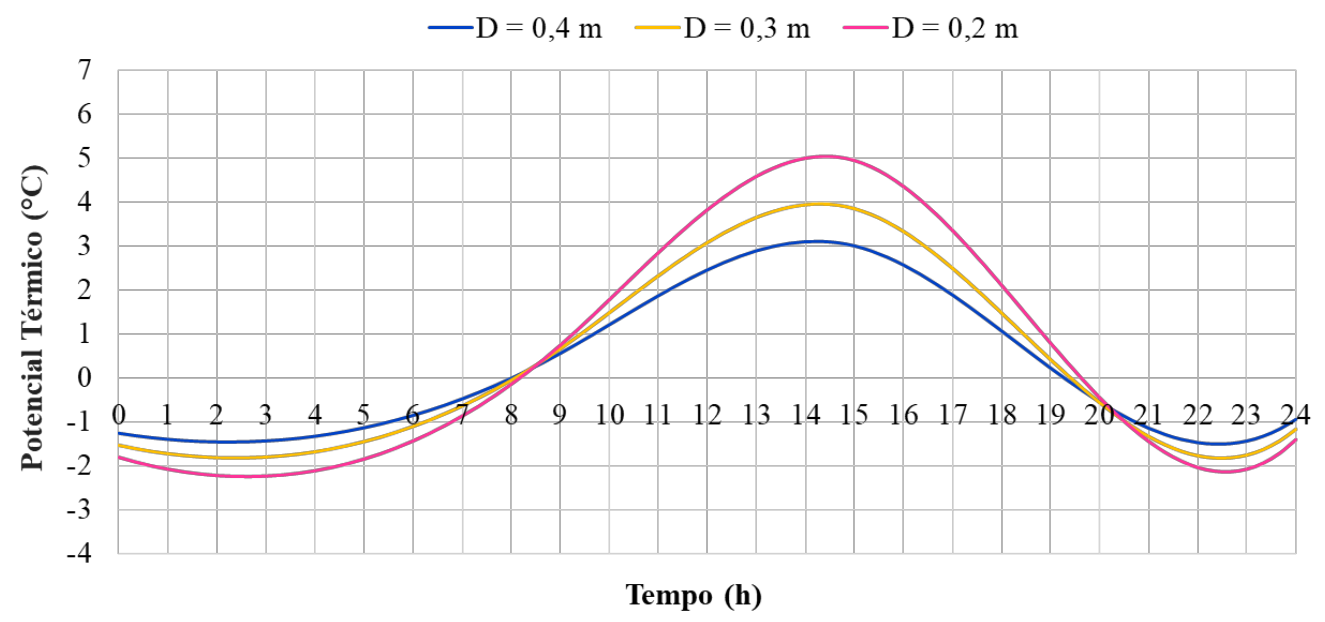

Finalmente, da Tabela 5, é determinado que à medida que o diâmetro do duto aumenta de $0,2 \mathrm{~m}$ para $0,3 \mathrm{~m} \mathrm{e}$ de $0,2 \mathrm{~m}$ para $0,4 \mathrm{~m}$, o desempenho energético diário também aumenta em 133,02\% e 277,51\%, 89,44\% e $171,14 \%$, e $75,09 \%$ e $149,52 \%$, para velocidades de $0,5 \mathrm{~m} / \mathrm{s}, 1,25 \mathrm{~m} / \mathrm{s}$ e $2 \mathrm{~m} / \mathrm{s}$, respectivamente.

\section{Conclusões}

Através de um modelo numérico do sistema TCSA desenvolvido em ANSYS Fluent, o presente trabalho de pesquisa aborda o estudo da influência da velocidade do ar no comprimento do tubo, necessário para produzir uma queda específica na temperatura do ar externo. Além disso, o efeito da velocidade do escoamento do ar e o diâmetro do tubo no desempenho térmico nesse sistema também foram investigados. Este estudo revela que esses parâmetros têm uma influência considerável no desempenho térmico do sistema TCSA pelo qual devem ser levados em consideração ao projetar um sistema TCSA. Os principais resultados a seguir são extraídos do estudo.

O comprimento da posição do ponto do joelho diminui com a redução da velocidade do ar. Quando a velocidade do ar é de $0,5 \mathrm{~m} / \mathrm{s}$ uma queda de temperatura superior a $90 \%$ da redução total ao longo de todo o comprimento do trocador de calor é alcançada a $20 \mathrm{~m}$ da entrada, em comparação com os $35 \mathrm{~m}$ necessários para alcançar esse ponto a uma velocidade de $2 \mathrm{~m} / \mathrm{s}$.

O comprimento do tubo necessário para atingir uma queda específica na temperatura é reduzido usando velocidades de escoamento de ar menores. Uma redução de $5,399{ }^{\circ} \mathrm{C}$ da temperatura máxima do ar na entrada do duto é alcançada quando o ar viaja $20 \mathrm{~m}$ a uma velocidade de $0,5 \mathrm{~m} / \mathrm{s}$ em comparação com os 40

378 Vielma Vivas, G. A. V.; Guerra, D. R. S. 
$\mathrm{m}$ de comprimento necessários para atingir uma queda de $5,02{ }^{\circ} \mathrm{C}$ quando a velocidade do ar é de $2 \mathrm{~m} / \mathrm{s}$. Isso pode até certo ponto ajudar a reduzir o problema de grandes requisitos de área terrestre e grandes custos de escavação envolvidos na instalação de longos tubos no sistema TCSA.

Embora o aumento da velocidade do escoamento de ar (com um diâmetro específico do tubo) e do diâmetro do tubo (com uma velocidade específica do escoamento de ar) diminua o potencial térmico médio diário do TCSA, eles também aumentam a vazão mássica de ar e, portanto, aumentam a capacidade de resfriamento do sistema TCSA. Com o aumento na velocidade do ar de $0,5 \mathrm{~m} / \mathrm{s}$ para $2 \mathrm{~m} / \mathrm{s}$, o potencial térmico médio diário do TCSA, com diâmetros de $0,2 \mathrm{~m}, 0,3 \mathrm{~m}$ e $0,4 \mathrm{~m}$, é reduzido em $13,37 \%, 28,40 \%$, e $37,01 \%$, respectivamente, mas o desempenho energético diário do sistema aumenta $281,17 \%, 186,40 \%$ e $151,95 \%$, para os mesmos diâmetros. Da mesma forma, o aumento do diâmetro do duto de $0,2 \mathrm{~m}$ para $0,4 \mathrm{~m}$ produz uma redução no potencial térmico médio diário do TCSA, para velocidade do ar de $0,5 \mathrm{~m} / \mathrm{s}, 1,25 \mathrm{~m} / \mathrm{s}$ e 2 $\mathrm{m} / \mathrm{s}$, de $14,21 \%, 32,22 \%$ e $37,62 \%$, respectivamente, mas o desempenho energético diário do sistema aumenta $277,51 \%, 171,14 \%$ e $149,52 \%$, para as mesmas velocidades.

O potencial térmico instantâneo máximo pode ser aumentado reduzindo a velocidade do escoamento do ar e o diâmetro do duto. Com um diâmetro do duto de $0,2 \mathrm{~m}$ e uma velocidade do ar de $0,5 \mathrm{~m} / \mathrm{s}$, esse potencial foi de $5,91{ }^{\circ} \mathrm{C}$. Este resultado mostra o potencial do sistema TCSA para sua implementação na cidade de Belém.

\section{Referências}

AGRAWAL, K. K. et al. Effect of soil moisture contentes on thermal performance of earth-air-pipe heat exchanger for winter heating in arid climate: in situ measurement. Geothermics, v. 77, p. 12-23, $2019 \mathrm{~b}$.

AGRAWAL, K. K. et al. Experimental study to investigate the effect of water impregnation on thermal performance of Earth air tunnel heat exchanger for summer cooling in hot and arid climate. Renewable Energy, v. 120, p. 255-265, 2018.

AGRAWAL, K. K. et al. The state of art on the applications, technology integration, and latest research trends of earth-air-heat exchanger system. Geothermics, v. 82, p. 34-50, 2019a.

AGRAWAL, K. K.; MISRA, R.; AGRAWAL, G. D. To study the effect of different parameters on the thermal performance of ground-air heat exchanger system: In situ measurement. Renewable Energy, v. 146, p. 2070-2083, 2020.

ALVES, A. B. M.; SCHMID, A. L. Cooling and heating potential of underground soil according to depth and soil surface treatment in the Brazilian climatic regions. Energy and Buildings, v. 90, p. 41-50, 2015.

ASCIONE, F.; BELLIA, L.; MINICHIELLO, F. Earth-to-air heat exchangers for Italian climates.

Renewable Energy, v. 36, p. 2177-2188, 2011.

BANSAL, V. et al. Performance analysis of earth-pipe-air heat exchanger for summer cooling. Energy and Buildings, v. 42, p. 645-648, 2010.

BHARADWAJ, S. S.; BANSAL, N. K. Temperature distribution inside ground for various surface conditions. Building and Environment, v. 16, p. 183-192, 1981.

BISONIYA, T.S.; KUMAR, A.; BAREDAR, P. Experimental and analytical studies of earth- air heat exchanger (EAHE) systems in India: a review. Renewable and Sustainable Energy Reviews, v. 19, p. 238246, 2013.

BRUM, R. S. et al. A new computational modeling to predict the behavior of earth-air heat exchangers. Energy and Building, v. 64, p. 395-402, 2013.

BRUM, R. S. et al. Design evaluation of earth-air heat exchangers with multiple ducts. Renewable Energy, 2018.

BRUM, R. S. et al. Development of simplified numerical model for evaluation of the influence of soil-air heat exchanger installation depth over its thermal potential. International Journal of Advanced Renewable Energy Research, 1, p. 505-514, 2012.

BRUM, R. S. et al. On the design of two EAHE assemblies with four ducts. Defect and Diffusion Forum, v. 372, p. 31-39, 2016a. 
BRUM, R. S. et al. Transient models to analyze the influence of the air velocity and ducts diameter on the performance of earth-air heat exchangers. International Journal of Fluid Mechanics Research, v. 43, n. 56, p. 503-520, 2016 b.

CUNY, M. et al. Influence of coating soil types on the energy of earth-air heat exchanger. Energy and Building, v. 158, p. 1000-1012, 2018.

CUNY, M. et al. Influence of rainfall events on the energy performance of an earth-air heat exchanger embedded in a multilayered soil. Renewable Energy, v. 147. n. 2, p. 2664-2675, 2020.

ESTRADA, E. et al. The impact of latent heat exchanges on the design of earth air heat exchangers. Applied Thermal Engineering, v. 129, p. 306-317, 2018.

EUROPEAN CENTRE FOR MEDIUM-RANGE WEATHER FORECASTS. ERA-Interim/Land. Disponível em: https://www.ecmwf.int/en/forecasts/datasets/reanalysis-datasets/era-interim-land. Acesso em: 20 out. 2019.

INCROPERA, F. P. et al. Fundamentals of Heat and Mass Transfer. $6^{\text {th }}$. ed. Hoboken: John Wiley \& Sons, 2007.

INTERNATIONAL ENERGY AGENCY. The future of cooling: opportunities for energy efficient air conditioning. Paris, 2018.

INTERNATIONAL ENERGY AGENCY. Transition to sustainable buildings: strategies and opportunities to 2050. Paris, 2013.

ISSA, R.; LEITCH, K.; CHANG, B. Experimental heat transfer study on green roofs in a semiarid climate during summer. Journal of Construction Engineering, 2015.

JACOVIDES, C. P. et al. On the ground temperature applications profile for passive cooling in buildigs. Solar Energy, v. 57, p. 167-175, 1996.

KALOGIROU, S. A. Solar Energy Engineering. 2nd . ed. Boston: Academic Press, 2014.

LABORATÓRIO DE EFICIÊNCIA ENERGÉTICA EM EDIFICAÇÕES. Arquivos climáticos INMET 2018. Disponível em: http://www.labeee.ufsc.br/sites/default/files/arquivos_climaticos/2018.zip. Acesso em: 15 out. 2019.

LI, H. et al. Experimental investigation on the cooling performance of an Earth to Air Heat Exchanger (EAHE) equipped with an irrigation system to adjust soil moisture. Energy and Buildings, v. 196, p. 280292, 2019.

LIN, J. et al. Impact of soil moisture on the long-term energy performance of an earth-air heat exchanger system. Renewable Energy, v. 147. n. 2, p. 2676-2687, 2020.

LIU, Z. et al. Enhancing a vertical earth-to-air heat exchanger system using tubular phase change material. Journal of Cleaner Production, v. 237, 2019.

MEHDID, C. et al. Thermal design of Earth-to-air heat exchanger: part II a new transiente semi-analytical model and experimental validation for estimating air temperature. Journal of Cleaner Production, v. 198, p. 1536-1544, 2018.

MISRA, R. et al. CFD analysis based parametric study of derating factor for earth air tunnel heat exchanger. Applied Energy, v. 103, p. 266-277, 2013c.

MISRA, R. et al. Evaluating termal performance and energy conservation potential of hybrid earth air tunnel heat exchanger in hot and dry climate -In situ measurement. The Journal of Thermal Science and Engineering Applications, v. 147, n. 3, $2013 a$.

MISRA, R. et al. Transient analysis based determination of derating factor for Earth Air Tunnel Heat Exchanger in winter. Energy and Building, v. 58, p. 76-85, 2013b.

MORSHED, W. et al. Cooling performance of earth-to-air heat exchangers applied to a poultry barn in semi-desert areas of South Iraq. International Journal of Agricultural and Biological Engineering, v. 11, p. 47-53, 2018.

MOUMMI, N. et al. Refreshment by geothermal energy: theoretical and experimental study in the Biskra site (in French). Revue des Energies Renouvelables, v. 13, p. 399-406. 2010. 
NUNES, B. R. et al. Modelagem computacional aplicada ao estudo de um trocador de calor solo-ar com diferentes configurações geométricas. Scientia Plena, v. 11, n. 8, 2015.

OLIVEIRA, T. Análise comparativa de resultados de capacidade de suporte de estacas cravadas de concreto usando ensaios do tipo SPT e CPTU. Belém, 2018. Trabalho de Conclusão de Curso (Engenharia Civil) - Universidade Federal do Pará, Belém, 2018.

OMER, A. M. Ground-source heat pumps systems and applications. Renewable and Sustainable Energy Reviews, v.12, p. 344-371, 2008.

POPIEL, C.; WOJTKOWIAK, J.; BIERNACKA, B. Measurements of temperatura distribution in ground. Experimental Thermal and Fluid Science, v. 25, p. 301-309, 2001.

RAMALHO, J. V. A. et al. Fitting new constructal models for the termal potential of Earth-Air Heat Exchangers. Acta Scientiarum Technology, v. 40, p. 1-8, 2018.

RODRIGUES, M. K. et al. Estudo do potencial térmico de trocador de calor solo-ar em dois tipos de solos no município de rio grande (RS). Revista Brasileira de Energias Renováveis, v. 6, n. 3, p. 489-506, 2017.

RODRIGUES, M. K. et al. Numerical investigation about the improvement of the thermal potential of an earth-air heat exchanger (EAHE) employing the constructal design method. Renewable Energy, v. 80, p. 538-551, 2015.

RODRIGUES, M. K. et al. Thermal performance simulations of Earth-Air Heat Exchangers for different soils of a coastal city using in-situ data. Sustainable Energy Technologies and Assessments, v. 30, p. 224 229, 2018.

SONI, S.K.; PANDEY, M.; BARTARIA, V.N. Hybrid ground coupled heat exchanger systems for space heating/cooling applications: a review. Renewable and Sustainable Energy Reviews, v. 60, p. 724-738, 2016.

THE R PROJECT FOR STATISTICAL COMPUTING. Getting started. Disponível em: https://www.rproject.org/. Acesso em: 25 fev. 2020.

VAZ, J. et al. An experimental study on the use of Earth-Air Heat Exchangers (EAHE). Energy and Buildings, v. 72, p. 122-131, 2014.

VAZ, J. et al. Experimental and numerical analysis of na earth-air heat exchanger. Energy and Buildings, V. 43, p. 2476-2482, 2011.

VERSTEEG, H. K.; MALALASEKERA, W. An introduction to computational fluid dynamics: the finite volume method. $2^{\text {nd }}$. ed. England: Pearson Education Limited, 2007.

ZHOU, T. et al. Research on cooling performance of phase change material- filled earth-air heat exchanger. Energy Conversion and Management, v. 177, p. 210-223, 2018.

Giovanni Antonio Vielma Vivas

Programa de Pós-Graduação em Engenharia Mecânica | Universidade Federal do Pará | Rua Augusto Corrêa, 01, Labem, sala 3, Guamá | Belém - PA - Brasil | CEP 66075-110 | Tel.: (91) 98848-5090 | E-mail: gavv22@yahoo.com

Danielle Regina da Silva Guerra

Programa de Pós-Graduação em Engenharia Mecânica | Universidade Federal do Pará | Tel.: (91) 3201-7251 | E-mail: daguerra@ufpa.br

\section{Ambiente Construído}

Revista da Associação Nacional de Tecnologia do Ambiente Construído Av. Osvaldo Aranha, $99-3^{\circ}$ andar, Centro

Porto Alegre - RS - Brasil CEP 90035-190

Telefone: +55 (51) 3308-4084

Fax: +55 (51) 3308-4054

www.seer.ufrgs.br/ambienteconstruido

E-mail: ambienteconstruido@ufrgs.br 